\title{
Spinon Fermi surface spin liquid in a triangular lattice antiferromagnet $\mathrm{NaYbSe} 2$
}

\section{Peng-Ling Dai}

Beijing Normal University

\section{Gaoning Zhang}

ShanghaiTech University

\section{Yaofeng Xie}

Rice University https://orcid.org/0000-0003-4235-9188

\section{Chunruo Duan}

Rice University

\section{Yonghao Gao}

University of Hong Kong

\section{Zihao Zhu}

Fudan University

\section{Erxi Feng}

Oak Ridge National Laboratory

\section{Chien-Lung Huang}

Rice University https://orcid.org/0000-0002-9672-4315

\section{Huibo Cao}

Neutron Scattering Division, Oak Ridge National Laboratory, Oak Ridge, Tennessee 37831, USA https://orcid.org/0000-0002-5970-4980

\section{A. Podlesnyak}

Oak Ridge National Laboratory https://orcid.org/0000-0001-9366-6319

\section{Garrett Granroth}

Oak Ridge National Laboratory https://orcid.org/0000-0002-7583-8778

\section{S. Everett}

Oak Ridge National Laboratory

\section{Joerg Neuefeind}

Oak Ridge National Laboratory https://orcid.org/0000-0002-0563-1544

\section{David Voneshen}

ISIS Pulsed Neutron and Muon Source, Science and Technology Facilities Council https://orcid.org/0000-0001-5716-7184

\section{Shun Wang}

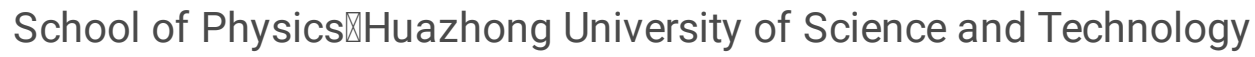

\section{Guotai Tan}


Department of Physics, Beijing Normal University https://orcid.org/0000-0001-8690-3574

\section{Emilia Morosan}

Rice University

\section{Xia Wang}

ShanghaiTech University

\section{Lei Shu}

Fudan University

\section{Gang Chen}

University of Hong Kong https://orcid.org/0000-0001-9339-6398

\section{Yanfeng Guo}

ShanghaiTech University, Shanghai 201210, China https://orcid.org/0000-0002-9386-4857

\section{Xingye Lu ( $\sim$ luxy@bnu.edu.cn )}

Beijing Normal University https://orcid.org/0000-0002-0409-1240

\section{Pengcheng Dai}

Rice University https://orcid.org/0000-0002-6088-3170

\section{Article}

Keywords: Quantum Spin Liquids, Spin Excitation Continuum, Neutron Scattering Measurements

Posted Date: October 5th, 2020

DOI: https://doi.org/10.21203/rs.3.rs-74447/v1

License: () (1) This work is licensed under a Creative Commons Attribution 4.0 International License. Read Full License 


\section{Spinon Fermi surface spin liquid in a triangular lattice antiferromagnet $\mathrm{NaYbSe}_{2}$}

Peng-Ling Dai\# ${ }^{1}$ Gaoning Zhang \# ${ }^{2}$ Yaofeng Xie, ${ }^{3}$ Chunruo Duan, ${ }^{3}$ Yonghao Gao, ${ }^{4}$ Zihao Zhu, ${ }^{4}$ Erxi Feng, ${ }^{5}$ Chien-Lung Huang, ${ }^{3}$ Huibo Cao, ${ }^{5}$ Andrey Podlesnyak, ${ }^{5}$ Garrett E. Granroth, ${ }^{5}$ Michelle S. Everett, ${ }^{5}$ Joerg C. Neuefeind, ${ }^{5}$ David Voneshen, ${ }^{6,7}$ Shun Wang, ${ }^{8}$ Guotai Tan, ${ }^{1}$ Emilia Morosan, ${ }^{3}$ Xia Wang, ${ }^{2}$ Lei Shu, ${ }^{4}$ Gang Chen, ${ }^{9,4}, *$ Yanfeng Guo, ${ }^{2, \dagger}$ Xingye Lu, ${ }^{1, \ddagger}$ and Pengcheng Dai ${ }^{3,}$ ${ }^{1}$ Center for Advanced Quantum Studies and Department of Physics, Beijing Normal University, Beijing 100875, China

${ }^{2}$ School of Physical Science and Technology, ShanghaiTech University, Shanghai 201210, China

${ }^{3}$ Department of Physics and Astronomy, Rice University, Houston, TX 77005, USA

${ }^{4}$ State Key Laboratory of Surface Physics, Department of Physics, Fudan University, Shanghai 200433, China

${ }^{5}$ Neutron Scattering Division, Oak Ridge National Laboratory, Oak Ridge, TN 37831

${ }^{6}$ ISIS Facility, Rutherford Appleton Laboratory, Chilton, Didcot, Oxfordshire OX11 OQX, UK

${ }^{7}$ Department of Physics, Royal Holloway University of London, Egham, TW20 OEX, UK

${ }^{8}$ School of Physics, Huazhong University of Science and Technology, Wuhan 430074, China

${ }^{9}$ Department of Physics and HKU-UCAS Joint Institute for Theoretical and Computational Physics at Hong Kong, The University of Hong Kong, Hong Kong, China

(Dated: September 9, 2020)

Triangular lattice of rare-earth ions with interacting effective spin- $1 / 2$ local moments is an ideal platform to explore the physics of quantum spin liquids (QSLs) in the presence of strong spin-orbit coupling, crystal electric fields, and geometrical frustration. The $\mathrm{Yb}$ delafossites, $\mathrm{NaYbCh}_{2}(\mathrm{Ch}=\mathrm{O}$, $\mathrm{S}$, Se) with $\mathrm{Yb}$ ions forming a perfect triangular lattice, have been suggested to be candidates for QSLs. Previous thermodynamics, nuclear magnetic resonance, and muon spin rotation powdersample neutron scattering measurements on $\mathrm{NaYbCh}_{2}$ have supported the suggestion of the QSL ground states. The key signature of a QSL, the spin excitation continuum, arising from the spin quantum number fractionalization, has not been observed. Here we perform both elastic and inelastic neutron scattering measurements as well as detailed thermodynamic measurements on high-quality single crystalline $\mathrm{NaYbSe}_{2}$ samples to confirm the absence of long-range magnetic order down to 40 $\mathrm{mK}$, and further reveal a clear signature of magnetic excitation continuum extending from 0.1 to 2.5 $\mathrm{meV}$. By comparing the structure of our magnetic excitation spectra with the theoretical expectation from the spinon continuum, we conclude that the ground state of $\mathrm{NaYbSe}_{2}$ is a QSL with a spinon Fermi surface.

Introduction. The quantum spin liquid (QSL) is a correlated quantum state in a solid where the spins 
of the unpaired electrons are highly entangled over long distances, yet they do not exhibit any long-range magnetic order in the zero temperature limit. Originally proposed by Anderson as the ground state for a system of $S=1 / 2$ spins on a two-dimensional (2D) triangular lattice that interact antiferromagnetically with their nearest neighbors [1], a QSL is a novel quantum state of matter beyond the traditional Landau's symmetry breaking paradigm [2-5], and might be relevant for our understanding of high-temperature superconductivity [6-8] and quantum computation in certain cases $[9,10]$. Beyond the simple characterization of absence of a magnetic order, one key signature of the excitations in a QSL is the presence of deconfined spinons that are fractionalized quasiparticles carrying spin-1/2, observed by inelastic neutron scattering as a spin excitation continuum fundamentally different from the integer spin wave excitations in an ordered magnet [11-16].

Although spin excitation continuum has been observed in the geometrically frustrated $S=1 / 2$ single crystal systems with 2D Kagomé [11], 2D triangular [12, 13], three-dimensional (3D) distorted Kagomé bilayers [14], and 3D pyrochlore $[15,16]$ lattices, there is no consensus on the microscopic origin of the observed spin excitation continuum. In the 2D $S=1 / 2$ Kagomé lattice $\mathrm{ZnCu}_{3}(\mathrm{OD})_{6} \mathrm{Cl}_{2}$ [11] and an effective $S=1 / 2$ triangular lattice magnet $\mathrm{YbMgGaO}_{4}[12,13]$, different interpretation of the observed spin excitation continuum includes a spin glass state of magnetic [17] and nonmagnetic $\mathrm{Mg}$-Ga site disorder due to intrinsic sample issues [18, 19], respectively, rather than the fractionalized quasiparticles of a QSL [5]. To conclusively identify the presence of deconfined spinon excitations in a QSL, one needs to search for the expected spin excitation continuum among candidate QSL materials with high quality single crystals and establish their physical properties with clear experimental signatures and structures.

Recently, geometrically frustrated 2D triangular-lattice rare-earth-based materials with effective $S=1 / 2$ local moments have attracted considerable attentions [20,21]. Compared with $\mathrm{YbMgGaO}_{4}[22]$, the family of $\mathrm{Yb}$ dichalcogenide delafossites $\mathrm{NaYbCh}_{2}(\mathrm{Ch}=\mathrm{O}, \mathrm{S}, \mathrm{Se})$ does not have the issue of $\mathrm{Mg}-\mathrm{Ga}$ site disorders in the non-magnetic layers and thus provides a genuine example for an interacting spin-1/2 triangular lattice antiferromagnet [23-25]. Moreover, $\mathrm{NaYbCh}_{2}$ exhibit larger magnetic anisotropy $\left(\frac{\Theta_{\mathrm{CW}, \mathrm{H} \perp \mathrm{c}}}{\Theta_{\mathrm{CW}, \mathrm{H} \| \mathrm{c}}}\right)$ than $\mathrm{YbMgGaO}_{4}$ (though the interlayer distance for $\mathrm{NaYbCh}_{2}$ is smaller), suggesting that the in-plane magnetic interactions play the dominant role. The combination of the strong spin-orbit coupling (SOC) and the crystal electric field (CEF) leads to a Kramers doublet ground state for the $\mathrm{Yb}^{3+}$ ion in $\mathrm{NaYbCh}_{2}$ that gives rise to the effective spin-1/2 local moment at each ion site. Since the energy gaps between the ground and first excited Kramers doublets CEF levels for $\mathrm{NaYbSe}_{2}$ [Fig. 1(b)] [26], $\mathrm{NaYbS}_{2}$ [24], and $\mathrm{NaYbO}_{2}$ [25] are well above $\sim 12 \mathrm{meV}$, the magnetic properties below $100 \mathrm{~K}$ can be safely interpreted from the interaction between the effective $S=1 / 2$ local moments. Although previous experiments on powder samples of $\mathrm{NaYbO}_{2}$ provided some positive evidence for QSL ground states [24, 27, 28], there are no detailed neutron 
scattering experiments on single crystalline samples to establish the presence of the magnetic excitation continuum and further reveal its wave vector, energy, temperature dependence. Here we report magnetic susceptibility, heat capacity, and neutron scattering results on single crystals of $\mathrm{NaYbSe}_{2}$. In addition to confirming the absence of long-range magnetic order down to $40 \mathrm{mK}$ and spin freezing down to $90 \mathrm{mK}$, we show the presence of a spin excitation continuum extending from 0.1 to $2.5 \mathrm{meV}$. Since our careful $\mathrm{X}$-ray diffraction structure refinement and pair-distribution function (PDF) analysis experiments reveal only $-4.8 \% \pm 1 \% \sim 5 \%$ of $\mathrm{Yb}$ on $\mathrm{Na}$ site and no evidence for a spin glass state at $40 \mathrm{mK}$, we conclude that the ground state of $\mathrm{NaYbSe}_{2}$ has signatures of a QSL, consistent with the expectation of a spinon Fermi surface quantum spin liquid state $[29,30]$.

Results. High quality single crystals of $\mathrm{NaYbSe}_{2}$ were grown by using flux method with $\mathrm{Te}$ as the flux (see Methods for further synthesis and experimental details). Figure 1(a) displays schematics of crystal structure and reciprocal space of $\mathrm{NaYbSe}_{2}$, where $\mathrm{Yb}$ ions form a perfect triangular lattice layer. Inelastic neutron scattering spectra of CEF excitations obtained by subtracting the scattering of $\mathrm{NaYbSe}_{2}$ from a nonmagnetic reference $\mathrm{NaYSe}_{2}$ is shown in Fig. 1(b) [29]. Consistent with previous work [26], the CEF levels of $\mathrm{Yb}^{3+}$ have a Kramers doublet ground state and three excited Kramers doublets at $E=15.7,24.5$, and $30.2 \mathrm{meV}$ at $T=13 \mathrm{~K}$, thus ensuring that all measurements below about $100 \mathrm{~K}$ can be safely considered as an effective $S=1 / 2$ ground state [26]. To characterize the behavior of the local moments of $\mathrm{Yb}$ and their exchange interactions, we measured the magnetic susceptibility of single-crystalline $\mathrm{NaYbSe}_{2}$. The temperature dependence of magnetization and the in-plane magnetic susceptibility $\chi_{\perp}(T)$ is depicted in Fig. 1(c), and a simple fit to the Curie-Weiss law yields $\Theta_{\mathrm{CW}, \perp} \simeq-13 \mathrm{~K}$ in the low-temperature region $(<20 \mathrm{~K})$, whose absolute value is larger than $\left|\Theta_{\mathrm{CW}, \perp}\right| \simeq 3.57 \mathrm{~K}$ when the Van Vleck contribution is subtracted [31], indicating the predominantly antiferromagnetic spin interactions in $\mathrm{NaYbSe}_{2}$. Heat capacity measurements were also performed to characterize the thermodynamics of $\mathrm{NaYbSe}_{2}$, and the pure magnetic contribution $C_{\text {mag }}(T)$ to the specific heat of $\mathrm{NaYbSe}_{2}$ and its dependence on applied magnetic fields from $0 \mathrm{~T}$ to $8 \mathrm{~T}$ are presented in Fig. 1(d). The data shows a broad peak that shifts upward in temperature as a function of increasing magnetic field for $\mathrm{H} \| c$, no sharp anomaly indicative of the onset of long-range order, consistent with the susceptibility result and earlier work [31]. Figure 1(e) also shows the estimated temperature dependence of $C_{\mathrm{mag}}(T) / T$ (left axis) and the corresponding magnetic entropy $S_{\text {mag }}$ (right axis). It is noted that $C_{\mathrm{mag}}(T) / T$ in the low-temperature regime $(<0.5 \mathrm{~K})$ is almost a constant, well compatible with the fact that the spinon Fermi surface alone has a constant density of states and would give a heat capacity depending linearly on temperature. Moreover, the temperature dependence of the magnetic entropy saturates to a value close to $S_{\mathrm{mag}} \approx R \ln 2$ (where $R$ is the ideal gas constant) around $15 \mathrm{~K}$, consistent with an effective spin- $1 / 2$ description of the $\mathrm{Yb}^{3+}$ local moment [31]. 
Although stoichiometric $\mathrm{NaYbSe}_{2}$ has no intrinsic structural disorder in the $\mathrm{Na}^{+}$intercalating layer [2325], real crystal could still have structural defects in $\mathrm{Na}^{+}$and $\mathrm{Se}^{2-}$ sites, and these vacant sites could be replaced by $\mathrm{Yb}^{3+}$ and $\mathrm{Te}^{2-}$, respectively (see Methods). To accurately determine the stoichiometry of our $\mathrm{NaYbSe}_{2}$, we carried out single crystal X-ray structural refinement by recording 1334 Bragg reflections, corresponding to 238 non-equivalent reflections. The Rietveld refinement results of the single-crystal X-ray diffraction data collected at $T=250 \mathrm{~K}$ are shown in Fig. 1(f) and the fitting outcome reveals full occupancy of the $\mathrm{Yb}^{3+}(3 a)$ and $\mathrm{Se}^{2-}(6 c)$ sites in the $\mathrm{YbSe}_{2}$ layers and $\sim 4.8 \% \pm 1 \%$ of the $\mathrm{Na}(3 b)$ sites occupied by the $\mathrm{Yb}$ ions. To further characterize the structural character of the sample, we have also performed PDF analysis on neutron diffraction data measured on 2.7 grams of $\mathrm{NaYbSe}_{2}$ powder ground from large amount of single crystals obtained from the same batches as the spin-excitation measurements. As shown in Fig. $1(\mathrm{~g})$, the local PDF peaks are well reproduced by fitting with the refined average structure using the X-ray diffraction data, indicating the absence of local distortion. The average structure includes a $\mathrm{Yb}$ substitution at the Na site and possible excess Te at the Se site. The PDF analysis suggests an upper limit of 10\% of $\mathrm{Yb}$ at the $\mathrm{Na}$ site and $0 \% \mathrm{Te}$ at the Se site. While this value is larger than that obtained by single crystal X-ray refinements, single crystal refinement results are more accurate as more Bragg peaks are measured in the X-ray refinements. These results are consistent with the inductively-coupled plasma measurements of chemical composition of the sample (see method for details). Although $\mathrm{Yb}$ ions in the Na layers may be magnetic, our frequency-dependent ac susceptibility measurements down to $90 \mathrm{mK}$ can be well described with a Curie-Weiss fit and show no evidence of spin freezing [Fig. 1(h)].

In the previous inelastic neutron scattering measurements on single crystals of $\mathrm{CsYbSe}_{2}\left(\Theta_{\mathrm{CW}} \simeq-13\right.$ $\mathrm{K})$, spin excitations were found to be centered around the $K$ point in reciprocal space [Fig. 1(a)], with no intensity modulation along the $c$-axis, and extending up to $1 \mathrm{meV}$ [32]. To determine what happens in $\mathrm{NaYbSe}_{2}$, we must first determine if the system has long/short-range magnetic order. For this purpose, we align the crystals in the $[H, H, 0] \times[0,0, L]$ and $[H, 0,0] \times[0, K, 0]$ zones [Fig. 1(a)]. Figures 2(a) and 2(b) display maps of elastic scattering in the $[H, H, L]$ and $[H, K, 0]$ planes, respectively, at $T=40 \mathrm{mK}$ (top panels) and $40 \mathrm{mK}-10 \mathrm{~K}$ (bottom panels). In both cases, no evidence of long/short magnetic order was observed at $40 \mathrm{mK}$, consistent with previous magnetic susceptibility, heat capacity, and nuclear magnetic resonance measurements [31]. The wave vector dependence of the spin excitations of $E=0.3 \pm 0.1 \mathrm{meV}$ in the $[H, H, L]$ zone at $40 \mathrm{mK}$ (left panel) and $10 \mathrm{~K}$ (right panel) is presented in Fig. 2(c). At $40 \mathrm{mK}$, one can see a featureless rod of scattering along the $[1 / 3,1 / 3, L]$ direction, indicating that spin excitations in $\mathrm{NaYbSe}_{2}$ are $2 \mathrm{D}$ in nature and have no $c$-axis modulations. The scattering essentially disappears at $10 \mathrm{~K}$, thus confirming the magnetic nature of the scattering at $40 \mathrm{mK}$. Moreover, Fig. 2(d) shows the temperature dependence of the $E=0.3 \pm 0.1 \mathrm{meV}$ spin excitations in the $[H, K, 0]$ zone. The magnetic scattering 
is centered around the $K$ point, consistent with the previous work [32], and decreases significantly with increasing temperature.

To further reveal the intrinsic quantum dynamics of the local moments of the $\mathrm{Yb}$ ions, we perform the inelastic neutron scattering measurements to study the spin excitations in single crystals of $\mathrm{NaYbSe}_{2}$ at both $40 \mathrm{mK}$ and $10 \mathrm{~K}$. Constant-energy images of spin excitations with a variety of energies in the inplane 2D Brillouin zones at $40 \mathrm{mK}$ and $10 \mathrm{~K}$ are summarized in Figs. 3(a-d) and 3(e-h), respectively. At $E=0.15 \pm 0.05 \mathrm{meV}$ and $40 \mathrm{mK}$, the magnetic scattering spectral weights spread broadly in the Brillouin zone but with higher intensity at the $K$ point and no scattering near the zone center (the $\Gamma$ point) [Fig. 3(a)]. This is clearly different from the wave vector dependence of the low-energy magnetic scattering for $\mathrm{YbMgGaO}_{4}$, in which the spectral weight is enhanced around the $M$ point [12]. The high intensity at the $K$ point in $\mathrm{NaYbSe}_{2}$ might arise from the strong $X Y$-type exchange interaction, since the strong $\mathrm{SOC}$ in this material indeed brings certain anisotropic interactions [33]. With increasing energies to $E=0.6 \pm 0.1$ [Fig. 3(b)], $1.1 \pm 1$ [Fig. 3(c)], and $E=2.1 \pm 0.1 \mathrm{meV}$ [Fig. 3(d)], the magnetic scattering spectral weights become more evenly distributed in the Brillouin zone and gradually decrease with increasing energy. While the spin excitation continuum at $E=0.15 \pm 0.05 \mathrm{meV}$ nearly vanishes on warming from $40 \mathrm{mK}$ to $10 \mathrm{~K}$ [Fig. 1(e)], the spectral weights at other energies become weaker but are still located around the Brillouin zone boundaries, especially the scattering at the $K$ points [Figs. 3(f-g)].

Figures 4(c) and 4(d) display the wave vector-energy dependence of the spin excitation spectral intensity (in log scale) along the magenta color arrow direction in Fig. 4(a) at $40 \mathrm{mK}$ and $10 \mathrm{~K}$, respectively. In both cases, the spectral intensity is broadly distributed in the energy-momentum plane, and the excitation intensity gradually decreases with increasing energy and finally vanishes above $\sim 2.2 \mathrm{meV}$. The broad neutron-scattering spectral intensity at $40 \mathrm{mK}$ persists to the lowest energy that we measured implying a high density of spinon scattering states at low energies. Moreover, the spectral weight around $\Gamma$ point is suppressed to form a V-shaped upper bound. Combining these two facts, it strongly suggests a spinon Fermi surface QSL since this scenario not only provides a high density of spinon states near the Fermi surface, but also well explains the V-shaped upper bound on the excitation energy near the $\Gamma$ point [30]. The $V$-shaped structure is one of the key properties for the magnetic excitation in the spinon Fermi surface quantum spin liquid. It arises from the large density of states and the linear $E-k$ spinon dispersion near the Fermi surface. Due to the spin quantum number fractionalization, the neutron scattering creates the spinon particle-hole pairs across the spinon Fermi surface. To excite the pair with an energy $E$, a minimal momentum transfer $E / v_{F}$ is needed where $v_{F}$ is the Fermi velocity. The slope of the V-shape is expected to be the Fermi velocity. It is also noted that the low-energy spin excitations clearly peak around the $K$ point at $40 \mathrm{mK}$ [Fig. 4(c)], and they decrease dramatically on warming but still keep the V-shaped upper bound around $\Gamma$ 
point at $10 \mathrm{~K}$ [Fig. 4(d)]. In addition, Figs. 4(e) and 4(f) present the wave vector-energy dependence of the spin excitation spectral intensity along the magenta color arrow directions in Fig. 4(b) at $40 \mathrm{mK}$ and $10 \mathrm{~K}$, respectively. The main results are similar to that in Figs. 4(c) and 4(d), and also support a spinon Fermi surface QSL.

The data points in Figs. 5(a) and 5(b) show energy dependence of spin excitations at the $K_{1}$ and $M_{2}$ points, respectively, under a variety of temperatures $T=40 \mathrm{mK}, 2 \mathrm{~K}$, and $10 \mathrm{~K}$. The solid lines in the figures display similar data at the $\Gamma_{1}$ point. Consistent with Fig. 4, magnetic scattering clearly decreases with increasing temperature at the $K_{1}$ and $M_{2}$ points, and essentially vanishes at the $\Gamma_{1}$ point. The temperature differences $(40 \mathrm{mK}-10 \mathrm{~K})$ of the imaginary part of the dynamic susceptibility, $\chi^{\prime \prime}(E)$, at the $K_{1}$ and $M_{2}$ points peak around 0.15 and $0.3 \mathrm{meV}$, respectively, as shown in the inset in Fig. 5(b). Besides, Fig. 5(c) compares energy dependence of the magnetic scattering at the $M_{1}, M_{2}$, and $K_{1}$ with the background at the $\Gamma_{2}$ point. To show the wave vector dependence of spin excitations, Figs. 5(d-g) plot the spectral intensity along the $[H, H, 0]$ direction for various energies of $E=0.25 \pm 0.1,0.5 \pm 0.1,1.3 \pm 0.1$, and $2.3 \pm 0.1 \mathrm{meV}$, respectively, at $T=40 \mathrm{mK}, 2 \mathrm{~K}$, and $10 \mathrm{~K}$. Similarly, Figs. 5(h) and 5(i) also plot constant-energy cuts along the $[0.5-K, 0.5+K, 0]$ direction for energies of $E=0.3 \pm 0.1,0.9 \pm 0.1,1.5 \pm 0.1,2.3 \pm 0.1 \mathrm{meV}$ at $40 \mathrm{mK}$ and $10 \mathrm{~K}$, respectively. All the results are compatible with Figs. 4(c-f). In Figs. 5(a)-5(b), the spin excitations can only be resolved above $E \sim 0.15 \mathrm{meV}$ because of the instrumental energy resolution. To further check whether the excitations are gapless, we show in Fig. 6 spin excitation energy spectra at $K_{1}$ point measured with improved instrumental energy resolution $\left(E_{i}=1.55 \mathrm{meV}\right)$. The energy dependent spin excitations for $T=40 \mathrm{mK}$ and $10 \mathrm{~K}$ reveals the persistence of the spin excitations down to $E \sim 0.06$ $\mathrm{meV}$, indicative of the gapless nature for the excitations.

Discussion and Conclusion. Overall, the magnetic and heat capacity measurements, combined with the neutron scattering results on single crystals of $\mathrm{NaYbSe}_{2}$ demonstrate the absence of long-range magnetic order even down to $40 \mathrm{mK}$, implying a quantum disordered QSL state. In particular, besides the naive disorder and the simple spectral continuum of spin excitation, the almost linear temperature dependence of magnetic heat capacity $C_{\mathrm{mag}}(T)$ at the low temperature regime, the enormous low energy gapless excitations and the V-shaped upper bound around the $\Gamma$ point in inelastic neutron scattering spectrum all strongly indicate the existence of a spinon Fermi surface. Theoretically, although the pure compact $U(1)$ gauge theory in two spatial dimensions is always confined due to the non-perturbative instanton events [34], it has been shown and understood that in the presence of spinon Fermi surface and gapless excitations, the QSL phase could be stable against gauge fluctuations, and a noncompact $U(1)$ gauge theory remains to be a good low energy description $[8,35]$. Therefore, our experimental results and conclusion about spinon Fermi surface QSL can be compatible with theory. The scenario of spinon Fermi surface QSL could further 
be verified by low temperature thermal transport measurement, which has an advantage to unveil the nature of low-energy itinerant excitations.

Very recently, the pressure-induced insulator to metal transition followed by an emergence of superconductivity in $\mathrm{NaYbSe}_{2}$ was observed in experiments [36]. This is quite remarkable since the QSL has long been thought to be a parent state of the high temperature superconductors [6-8]. It was suggested that doping a QSL could naturally result in superconductivity [6-8] due to the intimate relationship between high temperature superconductor and QSL, but the definitive experimental evidence showing that doping QSLs give rise to superconductivity is still lacking. Instead of doping, Ref. [36] obtained the superconductivity by pressure, which opens up a promising way to study the superconductivity in QSL candidates and sheds light on the mechanism of high temperature superconductivity.

* Electronic address: gangchen.physics@gmail.com

$\dagger$ Electronic address: guoyfeshanghaitech.edu.cn

\# Electronic address: luxy@bnu.edu.cn

$\S$ Electronic address: pdai@rice.edu

[1] Anderson, P. W. Resonating valence bonds: A new kind of insulator? Mater. Res. Bull. 8, 153 (1973).

[2] Balents, L. Spin liquids in frustrated magnets. Nature 464, 199-208 (2010).

[3] Zhou, Y., Kanoda, K. and Ng, T.-K. Quantum spin liquid states. Rev. Mod. Phys. 89, 025003 (2017).

[4] Savary, L. and Balents, L. Quantum spin liquids: a review. Rep. Prog. Phys. 80, 016502 (2017).

[5] Broholm, C. et al. Quantum spin liquids. Science 367, eaay0668 (2020).

[6] Anderson, P. W. The resonating valence bond state in $\mathrm{La}_{2} \mathrm{CuO}_{4}$ and superconductivity. Science 235, 1196-1198 (1987).

[7] Lee, P. A., Nagaosa, N. and Wen, X. G. Doping a Mott insulator: physics of high-temperature superconductivity. Rev. Mod. Phys. 78, 17-85 (2006).

[8] P. A. Lee, From high temperature superconductivity to quantum spin liquid: progress in strong correlation physics, Reports on Progress in Physics. 71, 012501 (2007).

[9] Kitaev, A. Y. Fault-tolerant quantum computation by anyons. Ann. Phys. 303, 2-30 (2003).

[10] Kitaev, A. Anyons in an exactly solved model and beyond. Ann. Phys. 321, 2-111 (2006).

[11] Han, T. H. et al. Fractionalized excitations in the spin-liquid state of a kagome-lattice antiferromagnet. Nature 492, 406-410 (2012).

[12] Shen, Y. et al. Evidence for a spinon Fermi surface in a triangular-lattice quantum-spin-liquid candidate, Nature (London) 540, 559 (2016).

[13] Paddison, J. A. M. et al. Continuous excitations of the triangular-lattice quantum spin liquid $\mathrm{YbMgGaO}_{4}$, $\mathrm{Nat}$ Phys. 13, 117 (2017). 
[14] Balz, C. et al. Physical realization of a quantum spin liquid based on a complex frustration mechanism. Nat. Phys. 12, 94217949 (2016).

[15] Gao, B. et al. Experimental signatures of a three-dimensional quantum spin liquid in effective spin-1/2 $\mathrm{Ce}_{2} \mathrm{Zr}_{2} \mathrm{O}_{7}$ pyrochlore. Nat. Phys. 15, 1052-1057 (2019).

[16] Gaudet, J. et al. Quantum spin ice dynamics in the dipole-octupole pyrochlore magnet $\mathrm{Ce}_{2} \mathrm{Zr}_{2} \mathrm{O}_{7}$. Phys. Rev. Lett. 122, 187201 (2019).

[17] Freedman, D. E. et al. Site specifc X-ray anomalous dispersion of the geometrically frustrated kagomé magnet, herbertsmithite, $\mathrm{ZnCu}_{3}(\mathrm{OH})_{6} \mathrm{Cl}_{2}$. J. Am. Chem. Soc. 132, 161851716190 (2010).

[18] Ma, Z. et al. Spin-glass ground state in a triangular-lattice compound $\mathrm{YbZnGaO}_{4}$, Phys. Rev. Lett. 120, 087201 (2018).

[19] Zhu, Z., Maksimov, P. A., White, S. R., and Chernyshev, A. L. Disorder-induced mimicry of a spin liquid in $\mathrm{YbMgGaO}_{4}$, Phys. Rev. Lett. 119, 157201 (2017).

[20] Rau, J. G. and Gingras, M. J. P. Frustration and anisotropic exchange in ytterbium magnets with edge-shared octahedra, Phys. Rev. B 98, 054408 (2018).

[21] Maksimov, P. A., Zhu, Z., White, S. R., and Chernyshev, A. L., Anisotropic-exchange magnets on a triangular lattice: spin waves, accidental degeneracies, and dual Spin Liquids, Phys. Rev. X 9, 021017 (2019).

[22] Li, Y. et al. Gapless quantum spin liquid ground state in the two-dimensional spin-1/2 triangular antiferromagnet $\mathrm{YbMgGaO}_{4}$, Sci. Rep. 5, 16419 (2015).

[23] Liu, W. et al. Rare-earth chalcogenides: A large family of triangular lattice spin liquid candidates, Chin. Phys. Lett. 35, 117501 (2018).

[24] Baenitz, M. et al. $\mathrm{NaYbS}_{2}$ : A planar spin-1/2 triangular-lattice magnet and putative spin liquid, Phys. Rev. B 98, 220409(R) (2018).

[25] Ranjith, K. M. et al. Field-induced instability of the quantum spin liquid ground state in the $J_{\text {eff }}=1 / 2$ triangular-lattice compound $\mathrm{NaYbO}_{2}$, Phys. Rev. B 99, 180401(R) (2019).

[26] Zhang, Z. et al. Crystalline Electric-Field Excitations in Quantum Spin Liquids Candidate $\mathrm{NaYbSe}_{2}$, arXiv:2002.04772.

[27] Bordelon, M. M. et al. Field-tunable quantum disordered ground state in the triangular-lattice antiferromagnet $\mathrm{NaYbO}_{2}$, Nat. Phys. 15, 1058 (2019).

[28] Ding, L. et al. Gapless spin-liquid state in the structurally disorder-free triangular antiferromagnet $\mathrm{NaYbO}_{2}$, Phys. Rev. B 100, 144432 (2019).

[29] See supplementary information for details.

[30] Li, Y. D., Lu, Y. M., and Chen, G., Spinon Fermi surface $U(1)$ spin liquid in the spin-orbit-coupled triangularlattice Mott insulator $\mathrm{YbMgGaO}_{4}$, Phys. Rev. B 96, 054445 (2017).

[31] Ranjith, K. M. et al. Anisotropic field-induced ordering in the triangular-lattice quantum spin liquid $\mathrm{NaYbSe}_{2}$, Phys. Rev. B 100, 224417(R) (2019).

[32] Xing, J. et al. Field-induced magnetic transition and spin fuctuations in the quantum spin-liquid candidate $\mathrm{CsYbSe}_{2}$, Phys. Rev. B 100, 220407(R) (2019). 
[33] Li, Y. and Chen, G., Detecting spin fractionalization in a spinon Fermi surface spin liquid, Phys. Rev. B 96, 075105 (2017).

[34] Polyakov, A. M., Quark confinement and topology of gauge theories, Nucl. Phys. B 120, 429 (1977).

[35] Lee, S. S., Stability of the $U(1)$ spin liquid with a spinon Fermi surface in $2+1$ dimensions, Phys. Rev. B 78, 085129 (2008).

[36] Jia, Y. et al. Mott Transition and Superconductivity in Quantum Spin Liquid Candidate $\mathrm{NaYbSe}_{2}$, arXiv:2003.09859, (2020).

[37] Rodríguez-Carvajal, J. Recent advances in magnetic structure determination by neutron powder diffraction. Phys. B 192, 55-69 (1993).

[38] Ehlers, G. et al. The new cold neutron chopper spectrometer at the Spallation Neutron Source: Design and performance, Rev. Sci. Instrum. 82, 085108 (2011).

[39] Abernathy, D. L. et al., Design and operation of the wide angular-range chopper spectrometer ARCS at the Spallation Neutron Source, Rev. Sci. Instrum. 83, 015114 (2012);

[40] Bewley, R. I. et al. LET, a cold neutron multi-disk chopper spectrometer at ISIS, Nuclear Instruments and Methods in Physics Research A 637, 128 (2011).

[41] Lu, X. et al.; (2020): Fractionalized magnetic excitations in a quantum spin liquid candidate $\mathrm{NaYbSe}_{2}$, $\mathrm{STFC}$ ISIS Neutron and Muon Source, https://doi.org/10.5286/ISIS.E.RB1920512.

[42] Arnold, O. et al. Mantid-Data analysis and visualization package for neutron scattering and $\mu$ SR experiments, Nuclear Instruments and Methods in Physics Research A 764, 156 (2014).

[43] Ewings, R. A. et al. HORACE: Software for the analysis of data from single crystal spectroscopy experiments at time-of-flight neutron instruments, Nuclear Instruments and Methods in Physics Research A 834, 132 (2016).

\section{Acknowledgments}

We thank M. Stone for suggestions of appropriate neutron scattering instrumentation and Feng Ye (ORNL) for the assistance with the single-crystal x-ray diffraction measurements. The research at Beijing Normal University is supported by the National Natural Science Foundation of China (Grant No. 11734002 and 11922402, X.L.). The work at ShanghaiTech university is supported by the National Natural Science Foundation of China (No. 11874264, Y.G.). Y.G. and X.W. thank the support from Analytical Instrumentation Center (\# SPST-AIC10112914), SPST, ShanghaiTech University. The neutron scattering work at Rice is supported by US DOE BES DE-SC0012311 (P.D.). This work is further supported by funds from the Ministry of Science and Technology of China ( grant No.2016YFA0301001, No.2018YFGH000095, No.2016YFA0300500 for G.C., and No.2016YFA0300501, No.2016YFA0300503 for L.S. and G.C.) and from the Research Grants Council of Hong Kong with General Research Fund Grant No.17303819 (G.C.). E.F. and H.C. acknowledges support of US DOE BES Early Career Award KC0402010 under Contract DE-AC05-00OR22725. E.M. and C.-L.H. acknowledge support from US DOE BES DE-SC0019503. This research used resources at Spallation Neutron Source, a DOE Office of Science User Facility operated by 
ORNL. We gratefully acknowledge the Science and Technology Facilities Council (STFC) for access to neutron beamtime at ISIS

\section{Author Contributions}

P.-L.D. and G.Z. contribute equally to this work. X.L., Y.G., and P.D. conceived this project. P.-L.D., G.T. and X.L. applied the beamtimes. G.Z. and Y.G. prepared the samples and did basic structure and magnetic characterizations with the help from S.W. and X.W.. E.F. and H.C. did X-ray structure refinement. Z.Z., C.-L.H., E.M. and L.S. performed the specific heat measurements. Z.Z. and L.S. analyzed the specific heat data. P.-L.D., X.L., Y.F.X. and C.D. performed neutron scattering experiments on CNCS, LET, and ARCS and NOMAD spectrometers with the help from A.P., D.V., G.E.G., M.S.E. and J.C.N.. P.-L.D. and X.L. analyzed the neutron scattering data and prepared the figures. Y.H.G. and G.C. provided the physical interpretation of the results. X.L., Y.H.G., G.C. and P.D. wrote the manuscript with input from Y.G.. All authors made comments.

\section{Competing interests}

The authors declare no competing interests.

\section{Methods}

Crystal Growth All the $\mathrm{NaYbSe}_{2}$ single crystals used in this study were grown by using $\mathrm{Te}$ as the flux. The starting materials are in a molar ration of $\mathrm{Na}: \mathrm{Yb}: \mathrm{Se}: \mathrm{Te}=1: 1: 2: 20$. To avoid the violent reaction between $\mathrm{Na}$ and $\mathrm{Se}$, the $\mathrm{Na}(99.7 \%)$ blocks and $\mathrm{Te}(99.999 \%)$ granules were mixed and slowly heated up to $200^{\circ} \mathrm{C}$ within 20 hours and pre-reacted at the temperature for 10 hours. The precursor was then thoroughly mixed with $\mathrm{Yb}(99.9 \%)$ blocks and Se (99.999\%) granules in the molar ratio and placed into an alumina crucible. The crucible was sealed into a quartz tube under the vacuum of $10^{-4} \mathrm{~Pa}$ and then slowly heated up to $950^{\circ} \mathrm{C}$ within 15 hours. After the reaction at this temperature for 20 hours, the assembly was slowly cooled down to $800^{\circ} \mathrm{C}$ at a temperature decreasing rate of $1{ }^{\circ} \mathrm{C} / \mathrm{h}$. At $800^{\circ} \mathrm{C}$, the quartz tube was immediately taken out of the furnace and placed into a high-speed centrifuge to separate the excess Te flux. To show a comparison, $\mathrm{NaYbSe}_{2}$ crystals were also grown by using $\mathrm{NaCl}$ as the flux in the similar procedure as mentioned above (not used for this study) [29]. The crystallographic phase and quality of the grown crystals were examined on a Bruker D8 VENTURE single crystal X-ray diffractometer using Mo $\mathrm{K}_{\alpha 1}$ radiation $(\lambda=0.71073 \AA)$ at room temperature. The crystals grown by using different flux have the same high quality [29]. Growth of the polycrystalline $\mathrm{NaYbSe}_{2}$ and $\mathrm{NaYSe}_{2}$ samples has been described elsewhere [31].

Stoichiometric Analysis The single crystal X-ray diffraction of $\mathrm{NaYbSe}_{2}$ were performed at $250 \mathrm{~K}$ on Rigaku XtaLAB PRO diffractometer at Spallation Neutron Source, ORNL. Structure refinement based on the X-ray diffraction data were carried out with FullProf suite [37], generating 
$\left(\mathrm{Na}_{0.952(10)} \mathrm{Yb}_{0.048(10)}\right) \mathrm{YbSe}_{2}$ without Te occupying Se sites. Elemental analysis of a group of $\mathrm{NaYbSe}_{2}$ single crystals grown with Te flux with a total mass of 35mg were performed by inductively-coupled plasma (ICP) method on Thermo Fisher ICP 7400 system. The result- $\mathrm{Na}_{0.965} \mathrm{Yb}_{1.03} \mathrm{Se}_{1.98} \mathrm{Te}_{0.025}$ - can be interpreted as $\sim 3 \%$ of $\mathrm{Na}^{+}$sites being occupied by $\mathrm{Yb}$ ions and agrees well with the structure refinement results of single-crystal x-ray diffraction, especially considering that Te could exist as flux in the sample.

Heat Capacity The specific heat capacity of $\mathrm{NaYbSe}_{2}$ was measured down to $50 \mathrm{mK}$ using a thermalrelaxation method in DynaCool-PPMS (Physical Property Measurement System, Quantum Design) with the magnetic field applied along the $c$-axis at Fudan University and Rice University. The total specific heat is described as a sum of magnetic and lattice contributions: $C_{p}=C_{\mathrm{mag}}+C_{\mathrm{phonon}}$. We fit the phonon contribution with $C_{\text {phonon }}=\beta T^{3}+\alpha T^{5}$.

Neutron Scattering The neutron scattering measurements of the magnetic excitations in $[\mathrm{H}, \mathrm{H}, \mathrm{L}]$ scattering plane, and the CEF excitations were performed on the Cold-Neutron-Chopper-Sepctrometer (CNCS) [38] and ARCS [39] at the Spallation Neutron Source (SNS), Oak-Ridge National Laboratory (ORNL), respectively. The measurements in $[\mathrm{H}, \mathrm{K}, 0]$ scattering plane were carried out on the LET cold neutron chopper spectrometer [40], ISIS spallation neutron source, Rutherford Appleton Laboratory (RAL), UK. We co-aligned $\sim 3.7$ grams of $\mathrm{NaYbSe}_{2}$ single crystals for the measurements of magnetic excitations and prepared $\sim 10$ grams $\mathrm{NaYbSe}_{2}$ and $\mathrm{NaYSe}_{2}$ polycrystalline samples for the CEF excitation measurements. The powder neutron diffraction experiment for pair-distribution function analysis were performed at NOMAD, ORNL at $100 \mathrm{~K}$, with 2.7 grams of $\mathrm{NaYbSe}_{2}$ polycrystalline sample ground from $\sim 100$ pieces of single crystals obtained from the same batches as the 3.7 gram sample set for our elastic/inelastic neutron scattering experiments at CNCS and LET. The neutron scattering data was reduced with Mantid [42] and analyzed with Mantidplot, Horace [43], and Mslice.

\section{Availability of Data and Codes}

The data as well as the codes used to analyze the data that support the plots in this paper and other findings of this study are available from the corresponding author on reasonable request. 

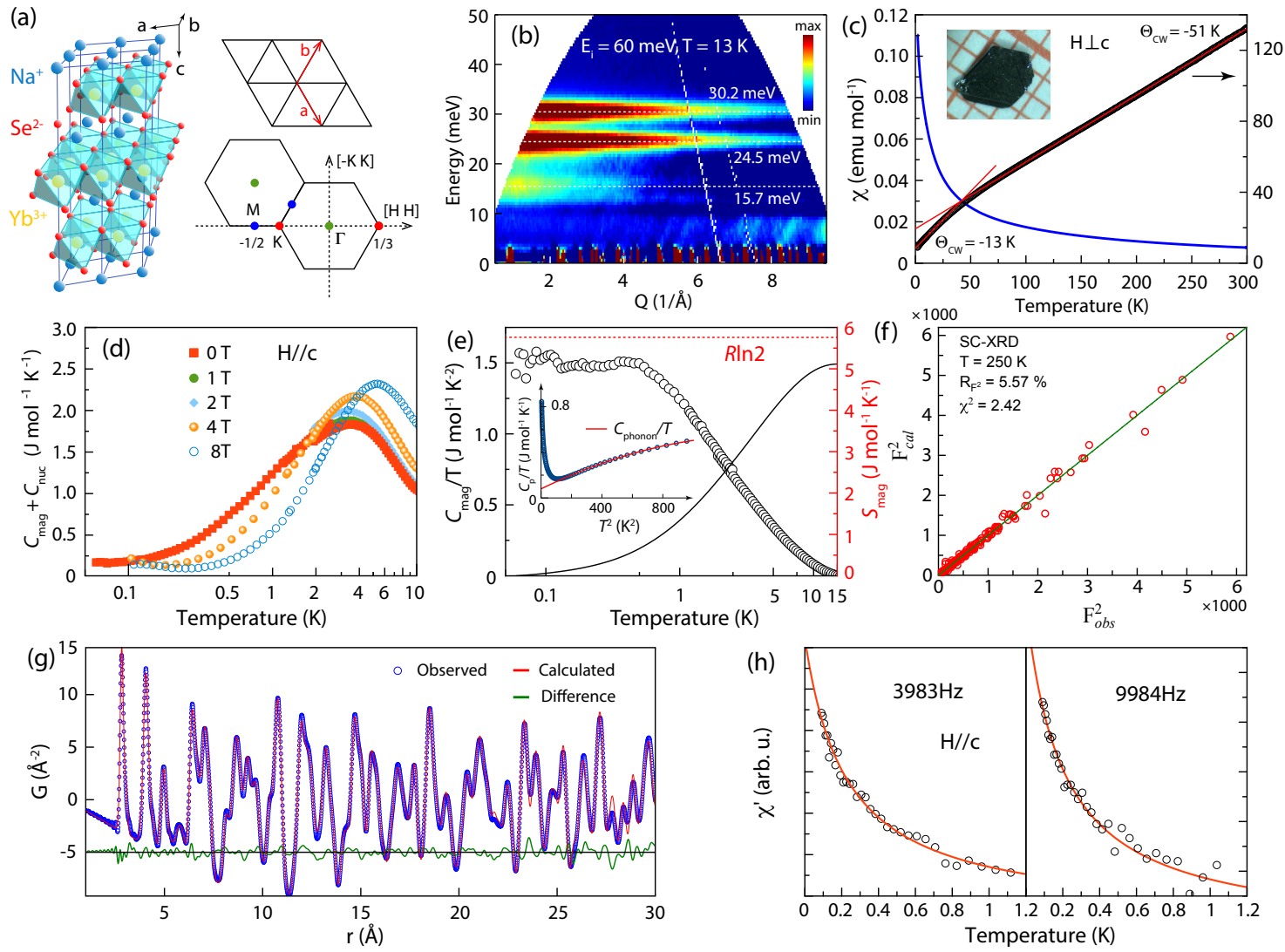

FIG. 1: Crystal structure and reciprocal space, CEF levels, heat capacity and stoichiometry of $\mathrm{NaYbSe}_{2}$. (a) The structure of $\mathrm{NaYbSe}_{2}$ and corresponding reciprocal space. The lattice parameters are $a=b \approx 4.07 \AA, c \approx 20.77$ $\AA$. (b) Inelastic neutron scattering spectra of CEF excitations obtained by subtracting the scattering of $\mathrm{NaYbSe}_{2}$ from a non-magnetic reference $\mathrm{NaYSe}_{2}$. Three CEF energy levels are marked by white dashed lines. (c) Temperaturedependent magnetization along $H \perp c$ direction. The fitting for high-temperature range $(\sim 160-300 \mathrm{~K})$ results in a Curie-Weiss temperature $\Theta_{\mathrm{CW}, \perp} \approx-51 \mathrm{~K}$, and the low temperature range $(<20 \mathrm{~K})$ generates a $\Theta_{\mathrm{CW}, \perp} \approx-13 \mathrm{~K}$. The inset shows the crystal for the magnetization measurements. (d) Temperature dependent specific heat $C_{\text {mag }}+C_{n u c}$ of $\mathrm{NaYbSe}_{2}$ and its dependence on applied magnetic fields $H \|$ c. $C_{\text {mag }}$ is magnetic contribution to the specific heat and $C_{\text {nuc }}$ arises from nuclear Schottky anomaly [29]. Phonon contribution has been subtracted. (e) Temperature dependent $C_{\mathrm{mag}} / T$ (black circle) with $C_{\text {nuc }} / T$ subtracted [29] and the magnetic entropy (black curve). The red dashed line marks the value of $R \ln 2$. The inset shows $C_{p} / T$ as a function of $T^{2}$. The red solid curve is a fitting of the phonon contribution $C_{\text {phonon }}$. (f) The Rietveld refinement results of the single-crystal X-ray diffraction data at $250 \mathrm{~K}$ yield $\mathrm{Na}_{0.952(10)} \mathrm{Yb}_{0.048(10)} \mathrm{YbSe}_{2} . \mathrm{F}_{c a l}^{2}$ and $\mathrm{F}_{\text {obs }}^{2}$ are the calculated and observed structure factors, respectively. (g) The PDF analysis of neutron data on $\mathrm{NaYbSe}_{2}$ up to $30 \AA$. The weighted residual value is $9.56 \%$. (h) AC susceptibility of $\mathrm{NaYbSe}_{2}$ single crystal measured with frequencies of $3983 \mathrm{~Hz}$ and $9984 \mathrm{~Hz}$. The red solid curves are Curie-Weiss fits for the data. 

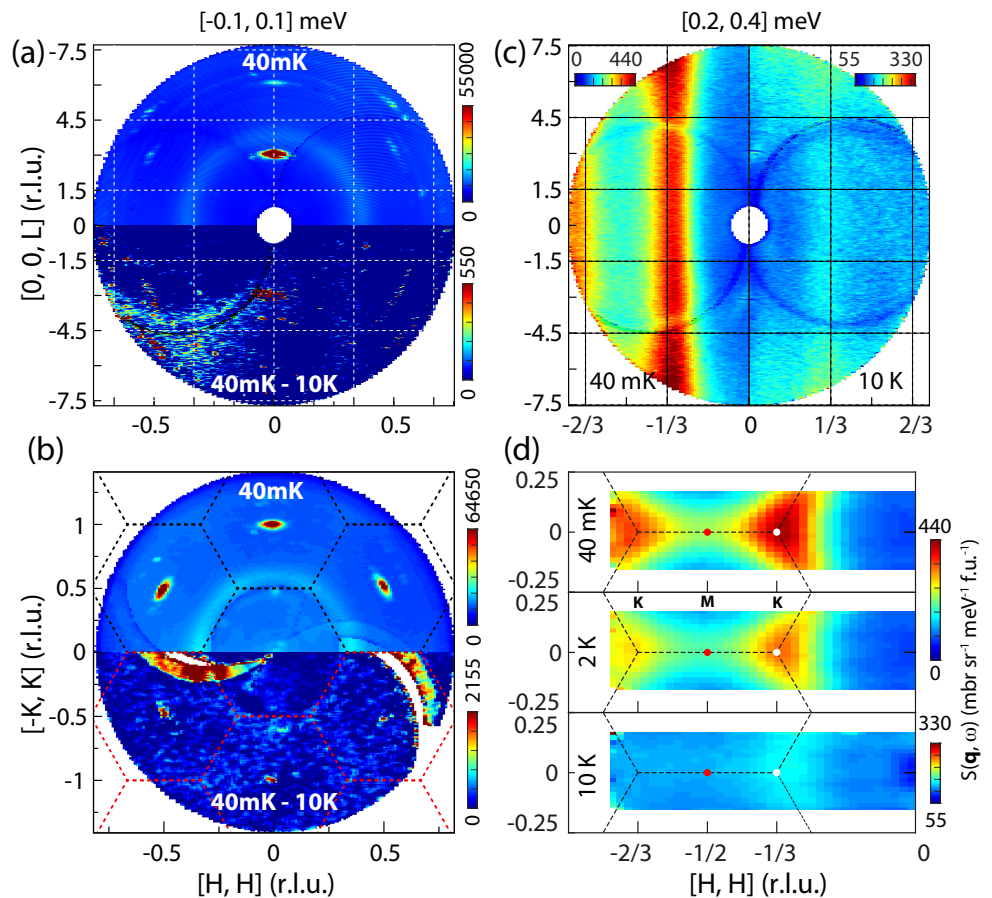

FIG. 2: Neutron scattering results in $[H, H, L]$ and $[H, K, 0]$ zones. Elastic neutron scattering results $(E=0 \pm 0.1$ $\mathrm{meV}$ ) in (a) the $[H, H, L]$ plane and (b) $[H, K, 0]$ plane measured with $E_{i}=3.32 \mathrm{meV}$ and $3.70 \mathrm{meV}$, respectively. Scattering along the vertical direction ( $[-K, K, 0]$ for (a) and $[0,0, L]$ for (b)) is integrated. The upper half panels of (a) and (b) are data at $T=40 \mathrm{mK}$, and the lower are the differences between $T=40 \mathrm{mK}$ and $10 \mathrm{~K}$. (c) $L$-dependence of the spin excitations along the $[H, H]$ direction at $T=40 \mathrm{mK}$ (left half panel) and $T=10 \mathrm{~K}$ (right half panel), with $K=[-0.05,0.05]$ and $E=0.3 \pm 0.1 \mathrm{meV}$. (d) Spin excitations with $E=0.3 \pm 0.1$ in the $[H, K]$ plane measured at $T=40 \mathrm{mK}, 2$, and $10 \mathrm{~K}$. Scattering along the $[0,0, L]$ direction is integrated. The black dashed lines mark the Brillouin zones of $\mathrm{NaYbSe}_{2}$. 

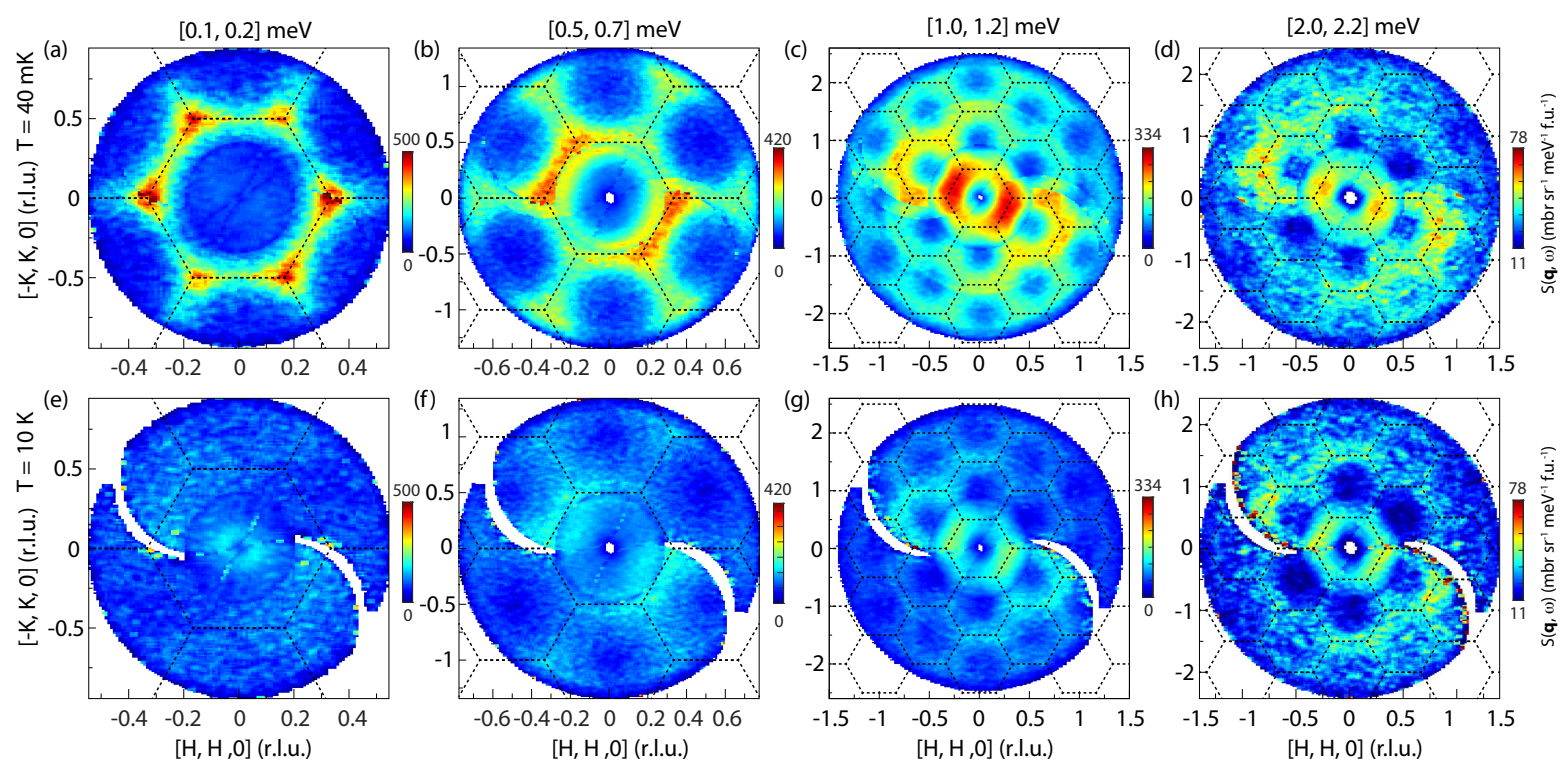

FIG. 3: Constant-energy images of spin excitations in the $[H, K, 0]$ plane. (a-d) Images at $T=40 \mathrm{mK}$ and (e-h) $10 \mathrm{~K}$. The intensity along the vertical $[0,0, L]$ direction is integrated. Spin excitations for (a,e) $E=0.15 \pm 0.05$, $(\mathrm{b}, \mathrm{f}) 0.6 \pm 0.1,(\mathrm{c}, \mathrm{g}) 1.1 \pm 0.1$, and $(\mathrm{d}, \mathrm{h}) 2.1 \pm 0.1 \mathrm{meV}$ are measured with $E_{i}=1.77,3.70,12.14$ and $12.14 \mathrm{meV}$, respectively. The black dashed lines mark the Brillouin zones in the reciprocal space. The data are collected in $180^{\circ}$ range of sample rotation around the $c$-axis. The $360^{\circ}$ circular coverage are generated by averaging the raw data and its mirror in the $[H, K, 0]$ plane. The $\mathrm{C}_{2}$-like anisotropy has been attributed to a trivial effect caused by sample-volume change in beam during sample rotation for neutron scattering measurements in [H, K, 0] plane [29]. 

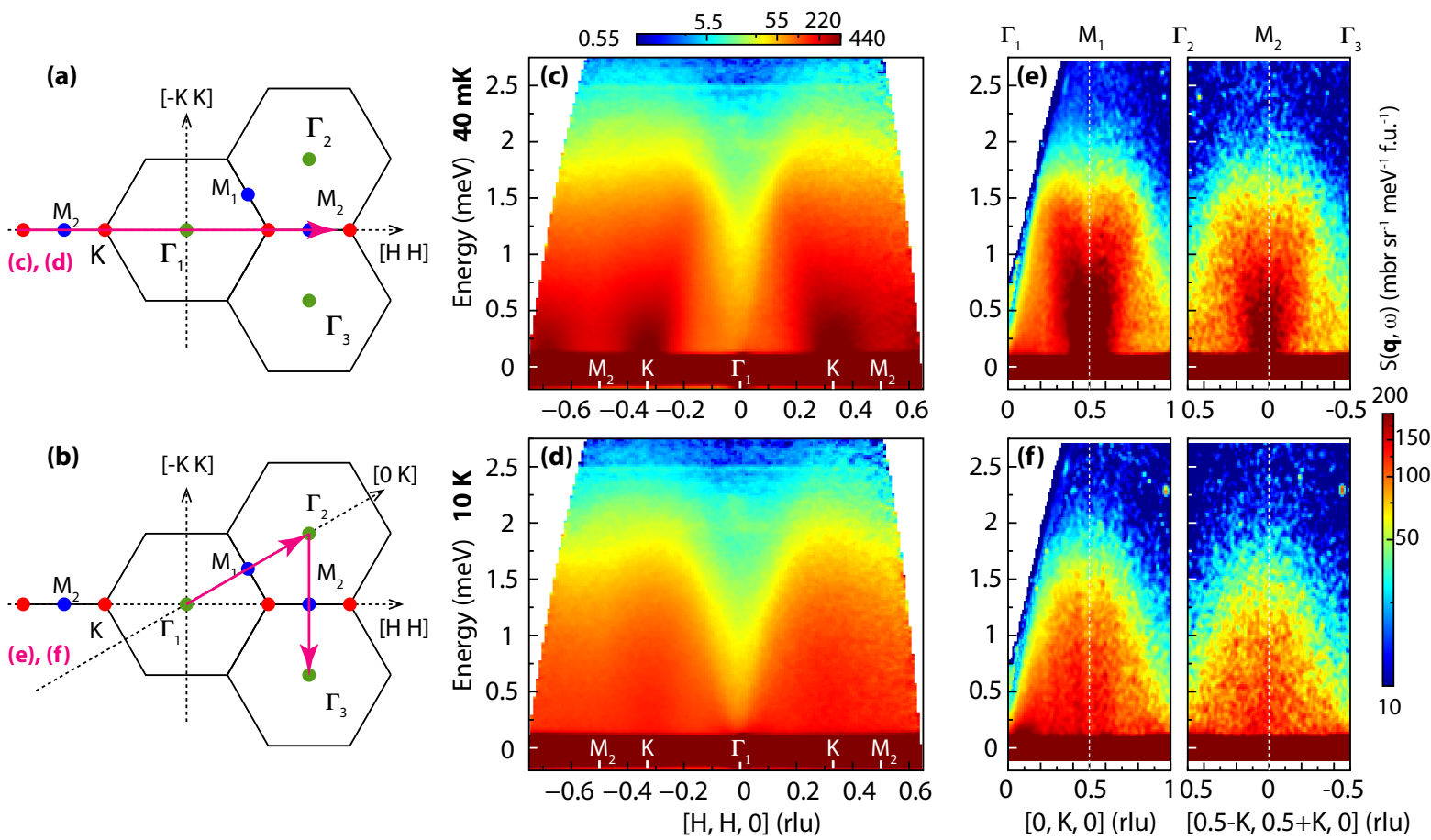

FIG. 4: Spin excitation spectra along high symmetry momentum directions. (a,b) Schematics of the Brillouin zones with high symmetry points $\Gamma, K$, and $M$ denoted by green, red, and blue dots, and the high symmetry directions for the images in (c-f) marked by pink lines with arrow heads. Spin excitation spectra collected at (c) $T=40 \mathrm{mK}$ and (d) $10 \mathrm{~K}$ along the $M_{2}-K-\Gamma-K-M_{2}$ with $E_{i}=3.32 \mathrm{meV}$. (e,f) Intensity color maps along the $\Gamma_{1}-M_{1}-\Gamma_{2}$ and $\Gamma_{2}-M_{2}-\Gamma_{3}$ directions measured with $E_{i}=3.7 \mathrm{meV}$. 

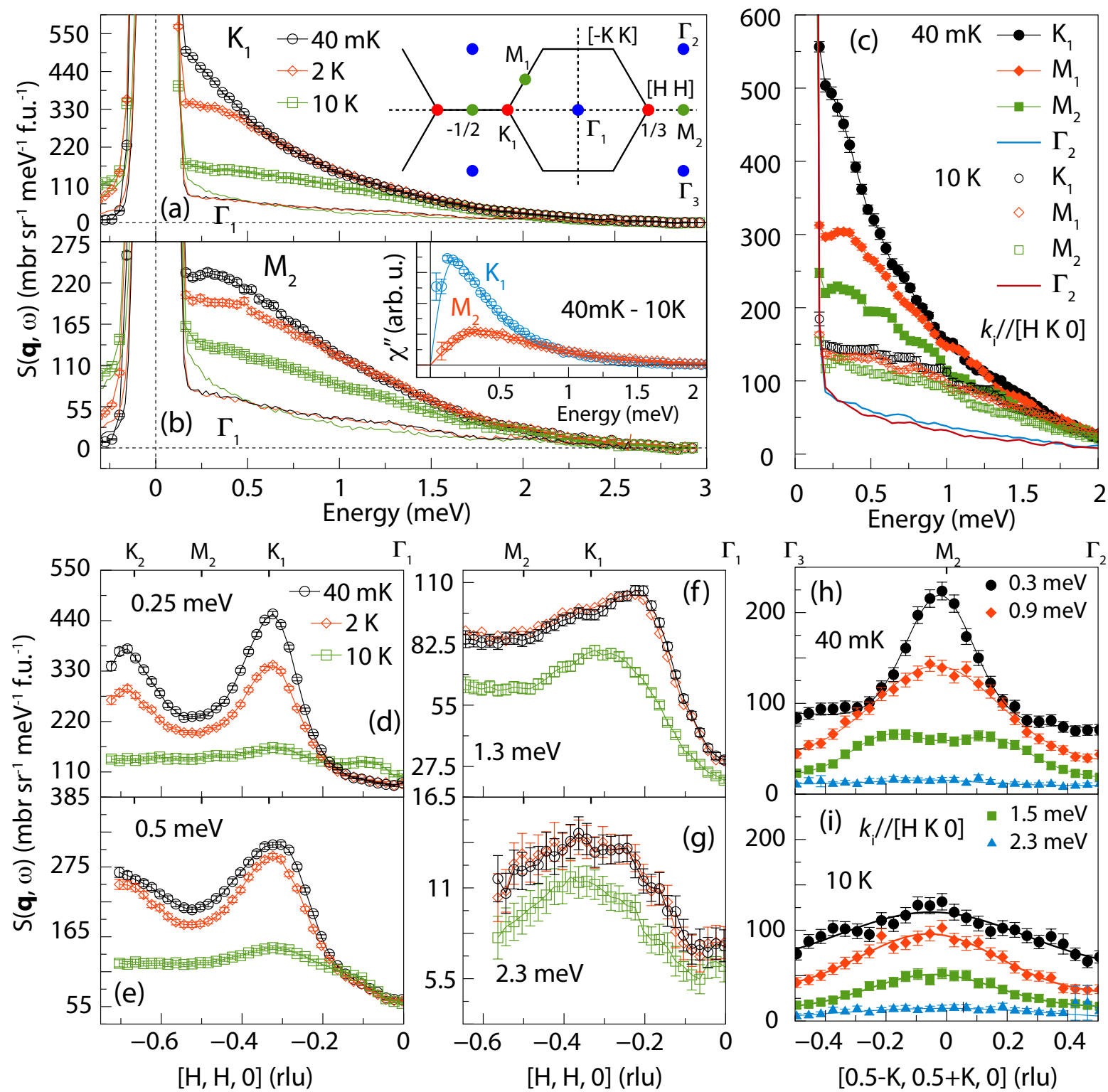

FIG. 5: Wave vector dependence of spin excitations along high symmetry directions. The wave vector cuts in (a,b,d-g) were measured in the $[H, H, L]$ zone with $E_{i}=3.32 \mathrm{meV}$, while those in (c,h,i) were measured in the $[H, K, 0]$ plane with $E_{i}=3.70 \mathrm{meV}$. (a) and (b) show the energy dependent scattering at $K_{1}$ and $M_{1}$ points measured at $T=40 \mathrm{mK}$ (black circle), $2 \mathrm{~K}$ (red diamond) and $10 \mathrm{~K}$ (green square). The inset in (a) is a schematic of the reciprocal space with the $\Gamma, K$ and $M$ points denoted by green, red and blue dots. The black, red, and green curves are energy cuts at $\Gamma_{1}$. The inset of (b) shows the difference of $\chi^{\prime \prime}$ between the spectra for $T=40 \mathrm{mK}$ and $10 \mathrm{~K}$ at the $K$ and $M$ points. The light blue and red curves are fittings of the $\chi^{\prime \prime}$ with a damped harmonic oscillator model. (c) shows the energy cuts at the $K_{1}, M_{1}, M_{2}$ and $\Gamma_{2}$. Solid symbols represent the data collected at $T=40 \mathrm{mK}$ and the open symbols collected at $10 \mathrm{~K}$. The black and blue curves are energy cuts at the $\Gamma_{2}$ point measured at $T=40$ $\mathrm{mK}$ and $10 \mathrm{~K}$. (d-g) Constant energy cuts along the $M_{2}-K_{1}-\Gamma_{1}$ for $T=40 \mathrm{mK}, 2 \mathrm{~K}$, and $10 \mathrm{~K}$, with corresponding energy transfers marked in the panels. Constant energy cuts along the $\Gamma_{3}-M_{2}-\Gamma_{2}$ measured at (h) $T=40 \mathrm{mK}$ and (i) $10 \mathrm{~K}$. The solid curves are guides to the eyes and the error bars represent one standard deviation. 


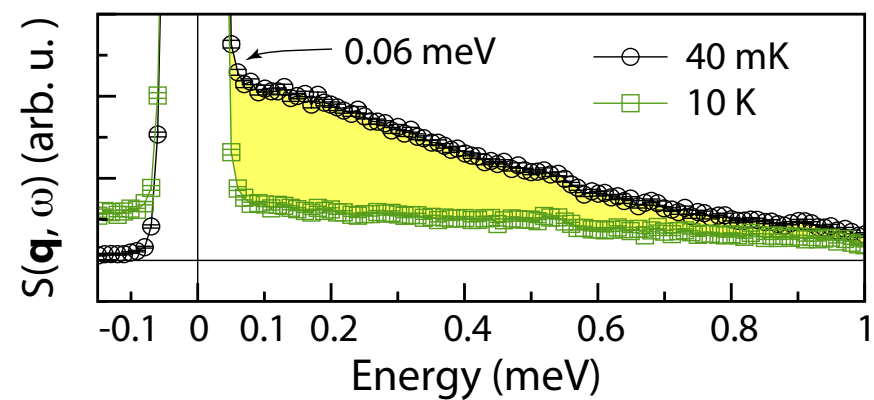

FIG. 6: Spin excitation energy spectra at $K_{1}$ position measured with $E_{i}=1.55 \mathrm{meV}$ at $T=40 \mathrm{mK}$ (red circles) and $10 \mathrm{~K}$ (green squares). The yellow shaded area marks the difference between the spectra for $T=40 \mathrm{mK}$ and $10 \mathrm{~K}$. The black arrow marks the lowest energy $(0.06 \mathrm{meV})$ magnetic excitations. 

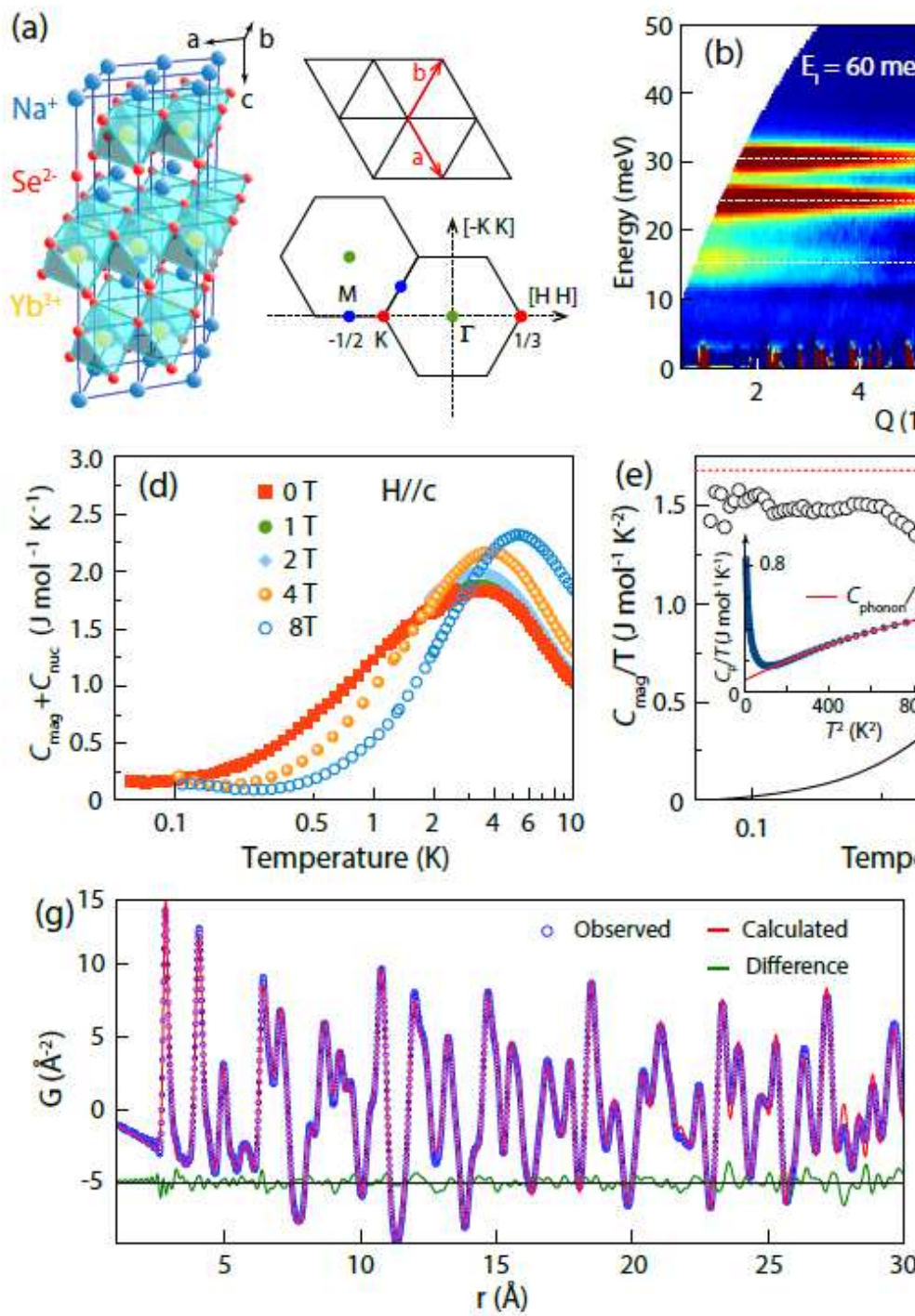
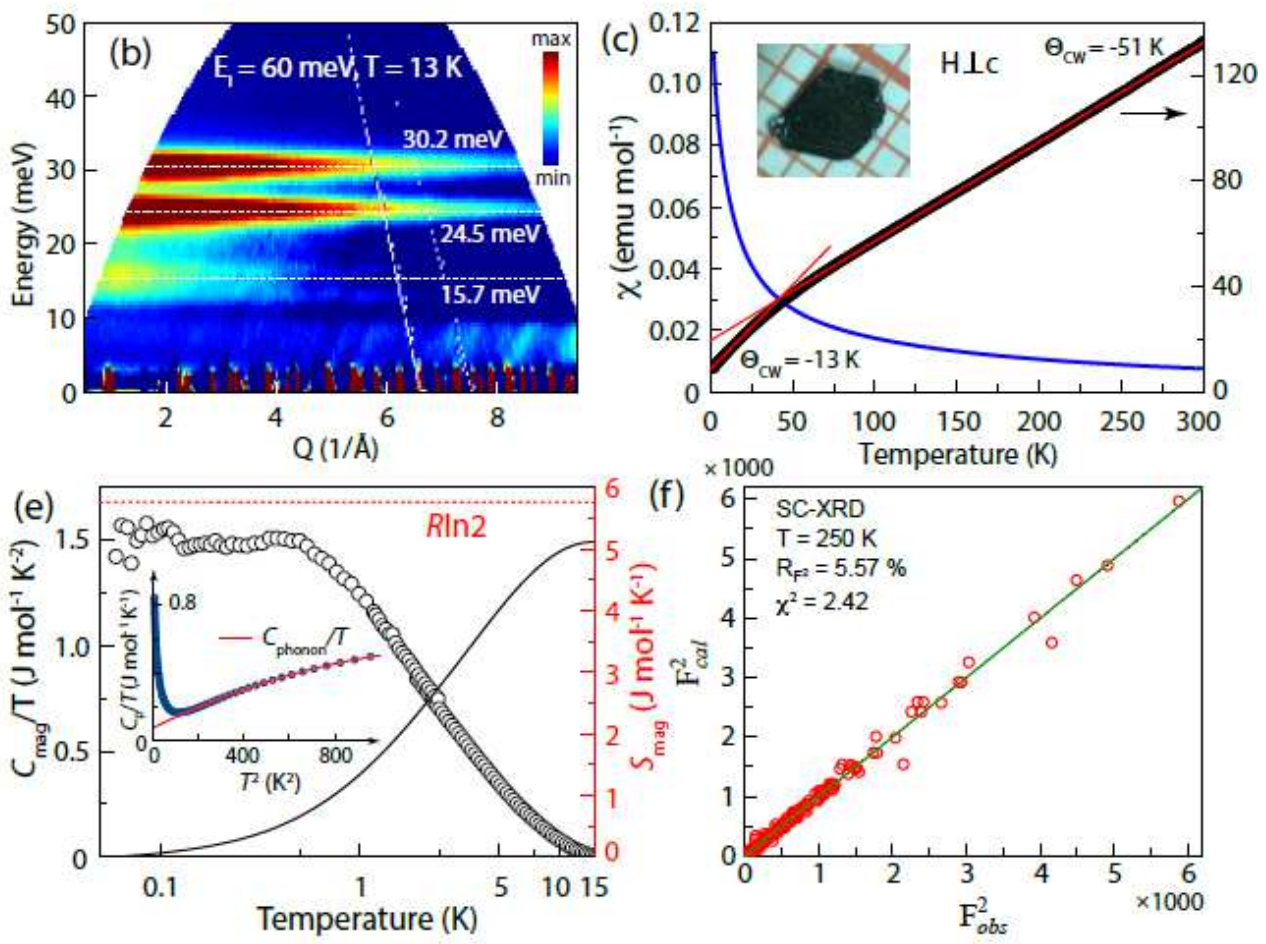

(f)
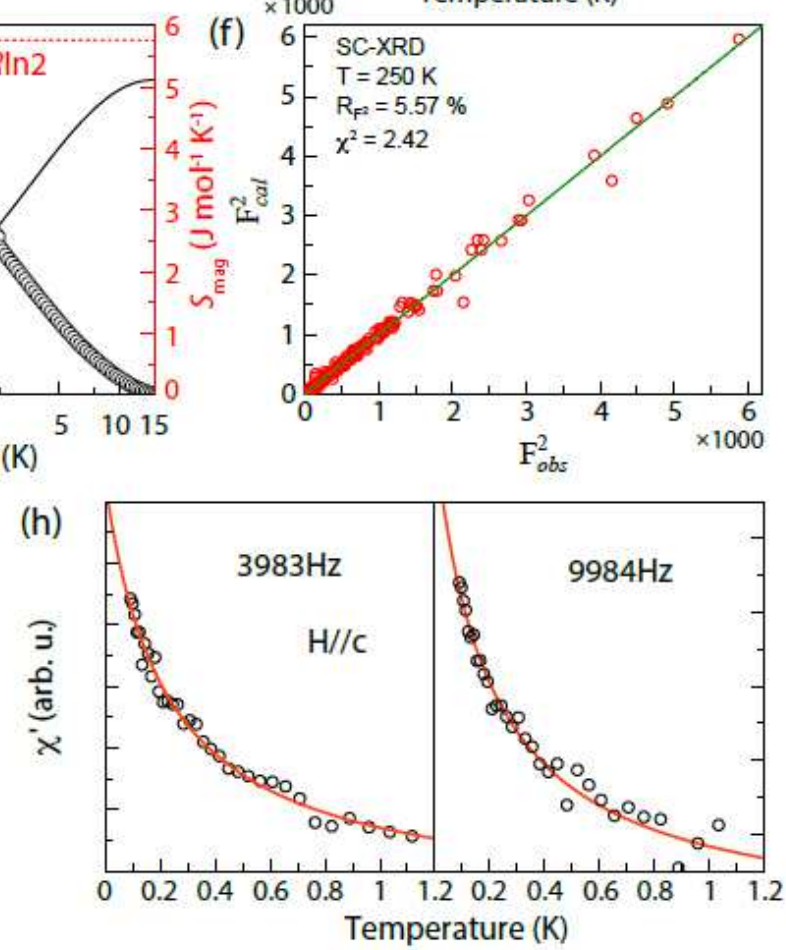

\section{Figure 1}

Crystal structure and reciprocal space, CEF levels, heat capacity and stoichiometry of NaYbSe2. (a) The structure of $\mathrm{NaYbSe} 2$ and corresponding reciprocal space. The lattice parameters are $a=b \approx 4.07 \AA, c \approx$ $20.77 \AA$. (b) Inelastic neutron scattering spectra of CEF excitations obtained by subtracting the scattering of $\mathrm{NaYbSe2}$ from a non-magnetic reference NaYSe2. Three CEF energy levels are marked by white dashed lines. (c) Temperature-dependent magnetization along $\mathrm{H} \otimes \mathrm{c}$ direction. The fitting for high-

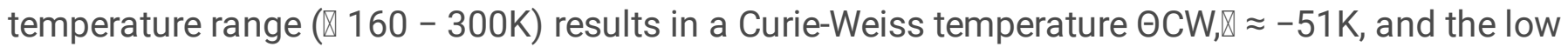
temperature range $(<20 \mathrm{~K})$ generates a $\Theta C W, \mathbb{Z} \approx-13 \mathrm{~K}$. The inset shows the crystal for the magnetization measurements. (d) Temperature dependent specific heat Cmag + Cnuc of NaYbSe2 and its dependence on applied magnetic fields $\mathrm{H} \otimes \mathrm{c}$. Cmag is magnetic contribution to the specific heat and Cnuc arises from nuclear Schottky anomaly [29]. Phonon contribution has been subtracted. (e) Temperature dependent Cmag/T (black circle) with Cnuc/T subtracted [29] and the magnetic entropy (black curve). The red dashed line marks the value of $R \ln 2$. The inset shows $C p / T$ as a function of $T 2$. The red solid curve is a 
fitting of the phonon contribution Cphonon. (f) The Rietveld refinement results of the single-crystal X-ray diffraction data at $250 \mathrm{~K}$ yield $\mathrm{Na0.952(10)Yb0.048(10)YbSe2.} \mathrm{F2cal} \mathrm{and} \mathrm{F2obs} \mathrm{are} \mathrm{the} \mathrm{calculated} \mathrm{and}$ observed structure factors, respectively. (g) The PDF analysis of neutron data on NaYbSe2 up to $30 \AA$. The weighted residual value is $9.56 \%$. (h) AC susceptibility of NaYbSe2 single crystal measured with frequencies of $3983 \mathrm{~Hz}$ and $9984 \mathrm{~Hz}$. The red solid curves are Curie-Weiss fits for the data.

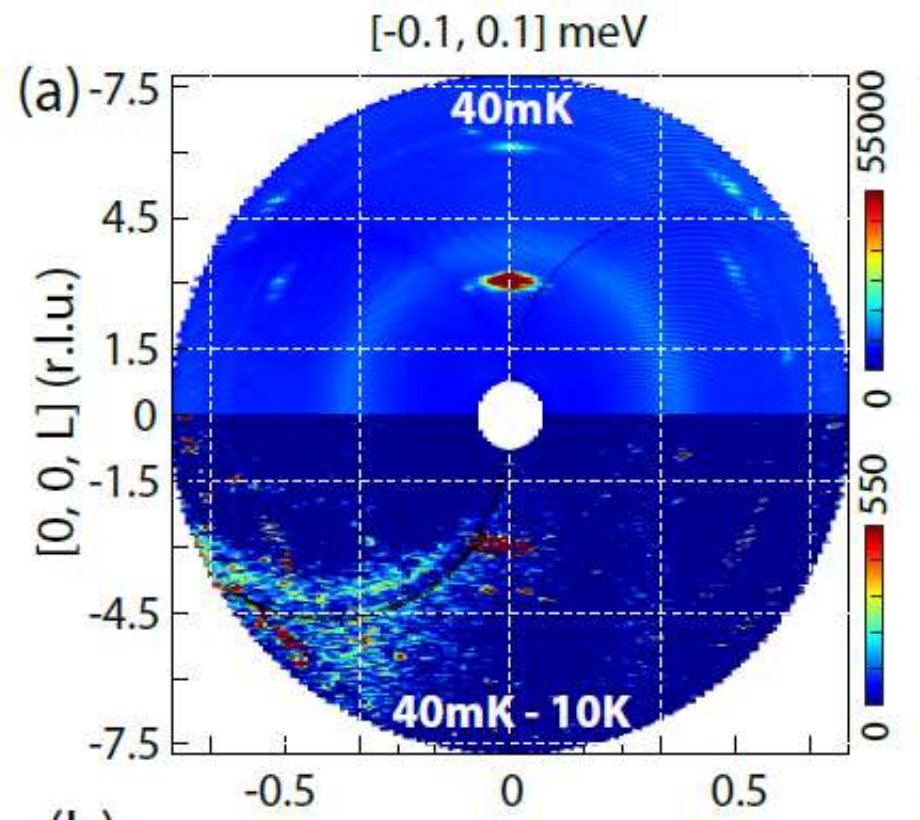

(b)
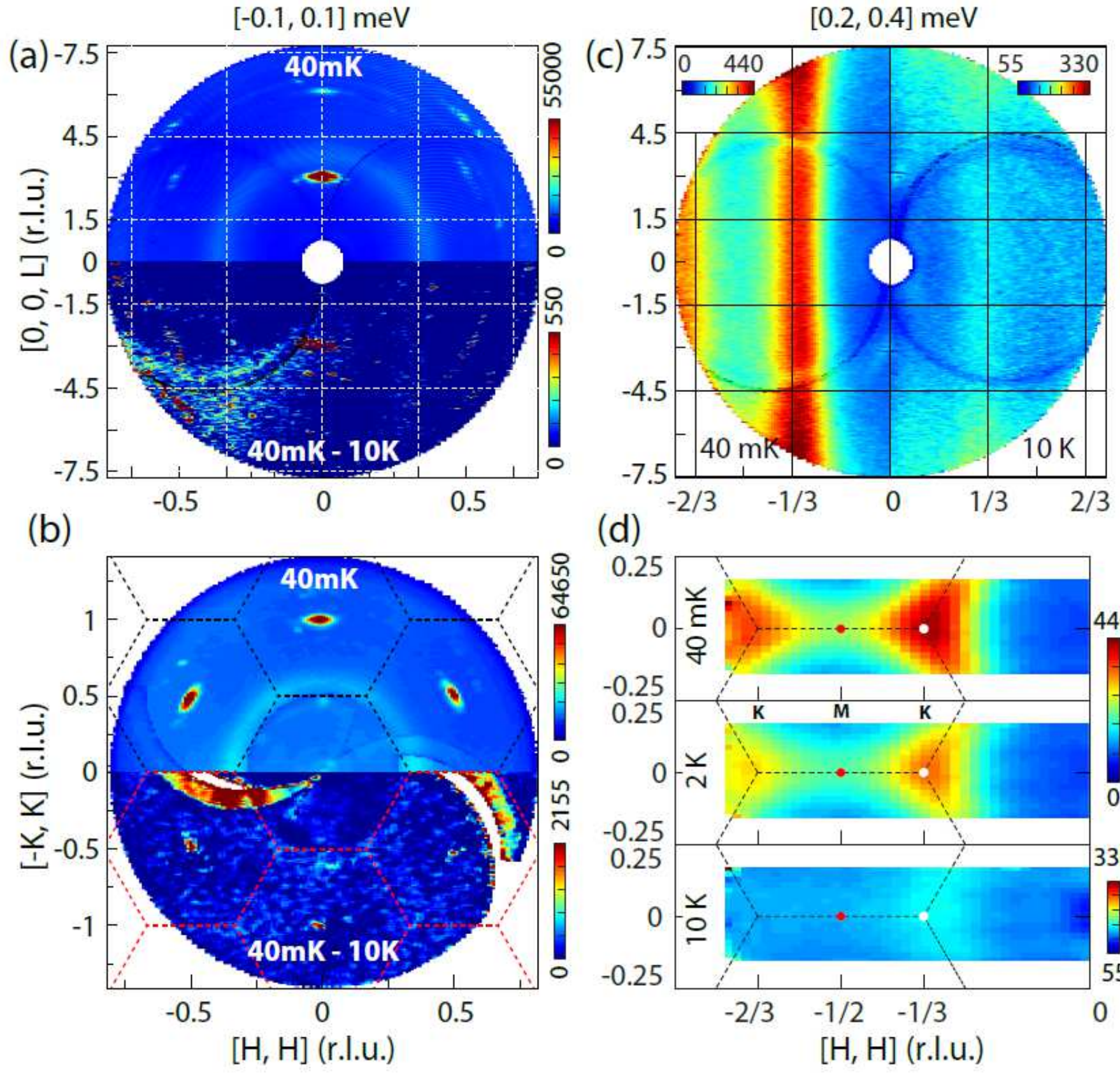

(d)

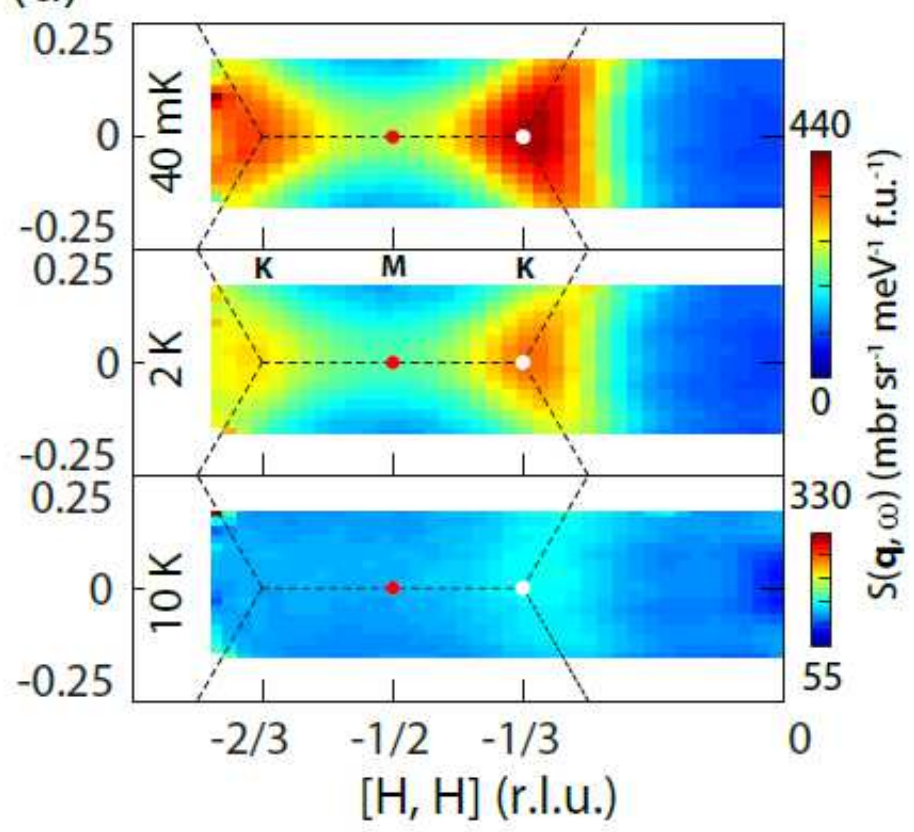

\section{Figure 2}

Neutron scattering results in $[\mathrm{H}, \mathrm{H}, \mathrm{L}]$ and $[\mathrm{H}, \mathrm{K}, 0]$ zones. Elastic neutron scattering results $(\mathrm{E}=0 \pm 0.1$ $\mathrm{meV})$ in (a) the $[\mathrm{H}, \mathrm{H}, \mathrm{L}]$ plane and $(\mathrm{b})[\mathrm{H}, \mathrm{K}, 0]$ plane measured with $\mathrm{Ei}=3.32 \mathrm{meV}$ and $3.70 \mathrm{meV}$, respectively. Scattering along the vertical direction $([-K, K, 0]$ for $(a)$ and $[0,0, L]$ for $(b))$ is integrated. The upper half panels of (a) and (b) are data at $\mathrm{T}=40 \mathrm{mK}$, and the lower are the differences between $\mathrm{T}=40$ $\mathrm{mK}$ and $10 \mathrm{~K}$. (c) L-dependence of the spin excitations along the $[\mathrm{H}, \mathrm{H}]$ direction at $\mathrm{T}=40 \mathrm{mK}$ (left half 
panel) and $\mathrm{T}=10 \mathrm{~K}$ (right half panel), with $\mathrm{K}=[-0.05,0.05]$ and $\mathrm{E}=0.3 \pm 0.1 \mathrm{meV}$. (d) Spin excitations with $E=0.3 \pm 0.1$ in the $[H, K]$ plane measured at $T=40 \mathrm{mK}, 2$, and $10 \mathrm{~K}$. Scattering along the $[0,0, \mathrm{~L}]$ direction is integrated. The black dashed lines mark the Brillouin zones of NaYbSe2.
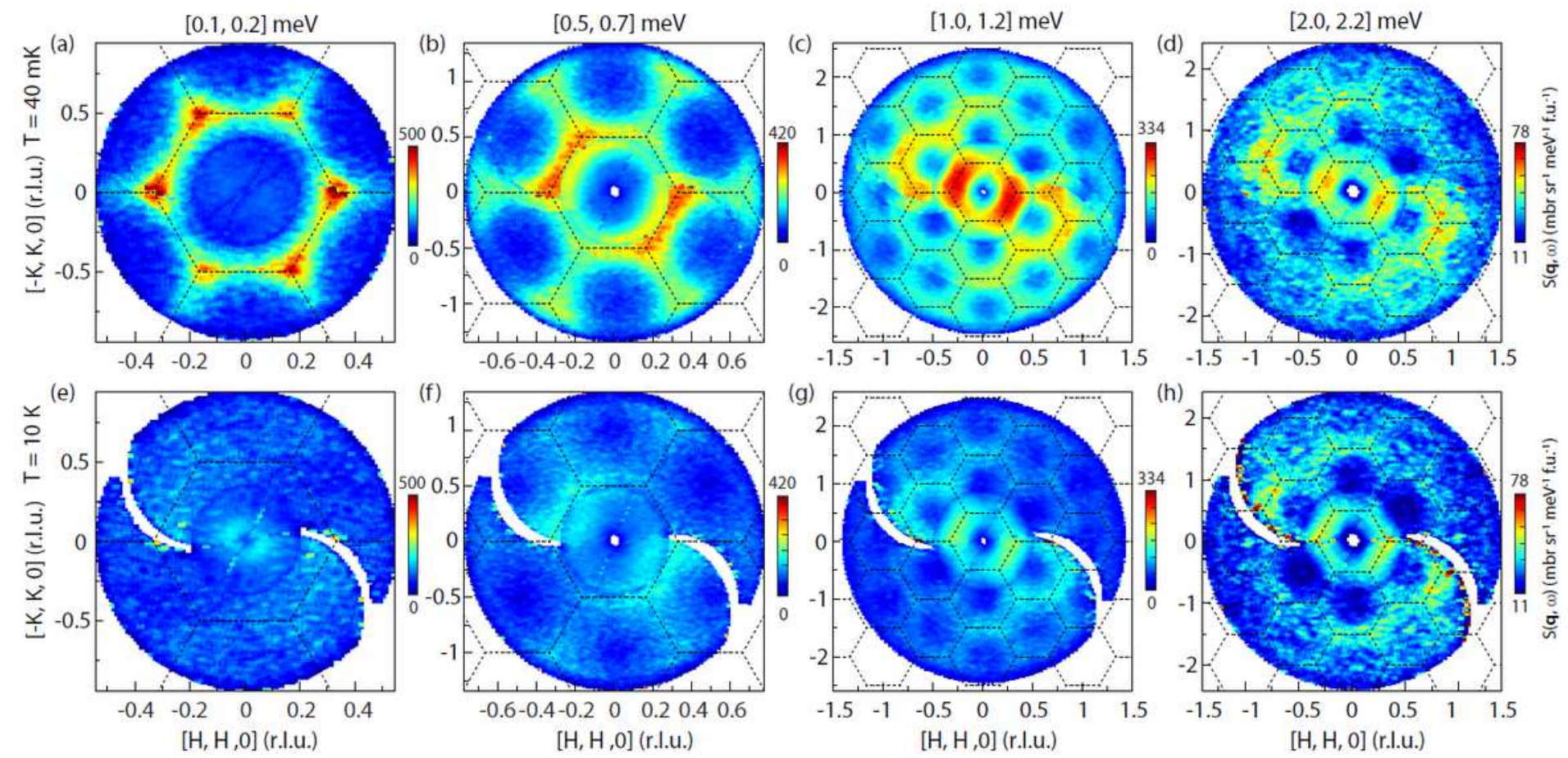

\section{Figure 3}

Constant-energy images of spin excitations in the $[\mathrm{H}, \mathrm{K}, 0]$ plane. (a-d) Images at $40 \mathrm{mK}$ and (e-h) $10 \mathrm{~K}$. The intensity along the vertical $[0,0$, L]direction is integrated. Spin excitations for $(a, e) E=0.15 \pm 0.05$, $(b, f)$ $0.6 \pm 0.1,(\mathrm{c}, \mathrm{g}) 1.1 \pm 0.1$, and $(\mathrm{d}, \mathrm{h}) 2.1 \pm 0.1 \mathrm{meV}$ are measured with $\mathrm{Ei}=1.77,3.70,12.14$ and $12.14 \mathrm{meV}$, respectively. The black dashed lines mark the Brillouin zones in the reciprocal space. The data are collected in $180 \rrbracket$ range of sample rotation around the c-axis. The $360 \rrbracket$ circular coverage are generated by averaging the raw data and its mirror in the $[\mathrm{H}, \mathrm{K}, 0]$ plane. The $\mathrm{C} 2$-like anisotropy has been attributed to a trivial effect caused by sample-volume change in beam during sample rotation for neutron scattering measurements in $[\mathrm{H}, \mathrm{K}, 0]$ plane [29]. 

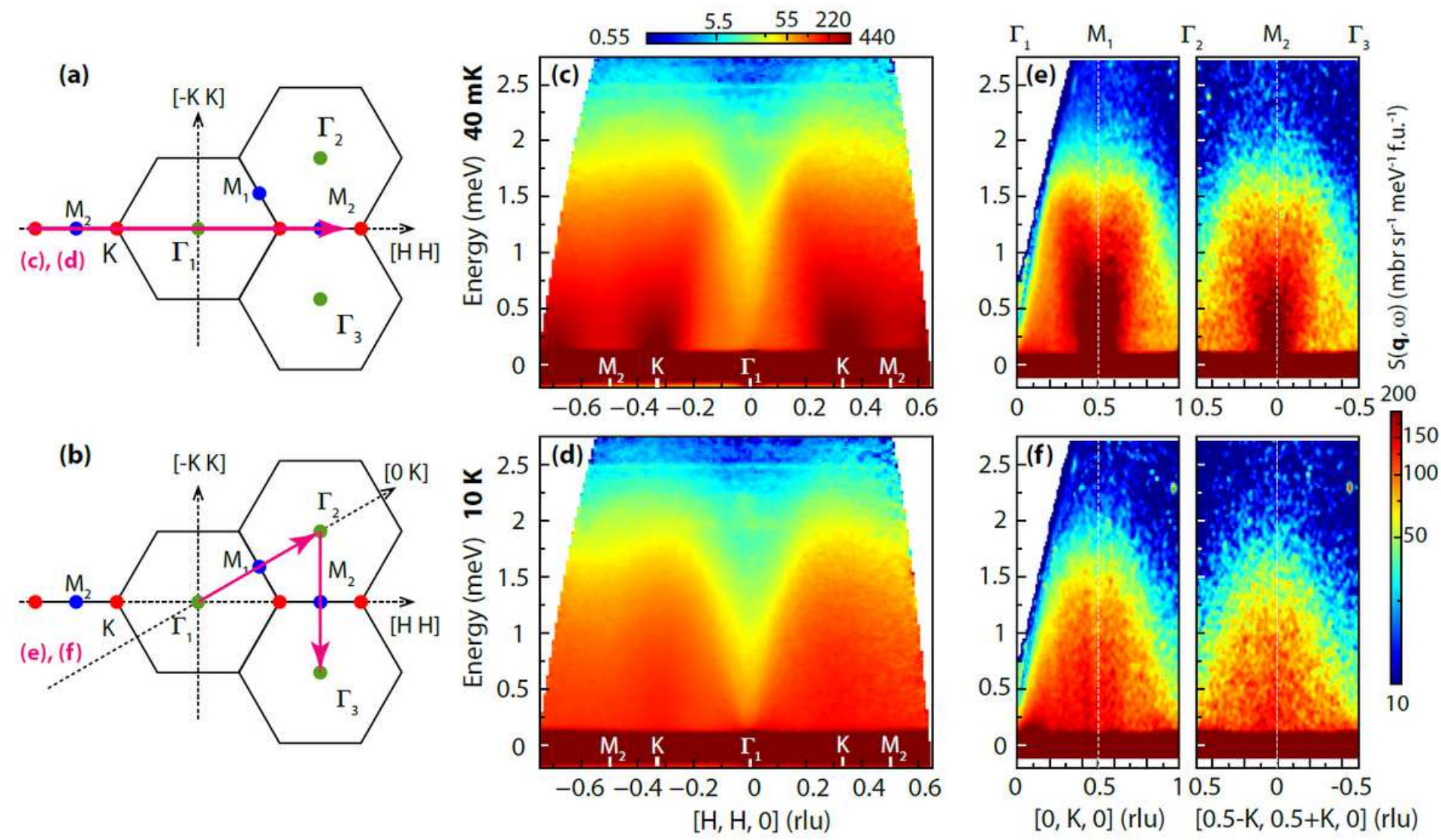

\section{Figure 4}

Spin excitation spectra along high symmetry momentum directions. $(a, b)$ Schematics of the Brillouin zones with high symmetry points $\Gamma, \mathrm{K}$, and $\mathrm{M}$ denoted by green, red, and blue dots, and the high symmetry directions for the images in (c-f) marked by pink lines with arrow heads. Spin excitation spectra collected at (c) $\mathrm{T}=40 \mathrm{mK}$ and (d) $10 \mathrm{~K}$ along the M2-K-Г-K-M2 with $\mathrm{Ei}=3.32 \mathrm{meV}$. (e,f) Intensity color maps along the Г1-M1-Г2 and Г2-M2-Г3 directions measured with $\mathrm{Ei}=3.7 \mathrm{meV}$. 

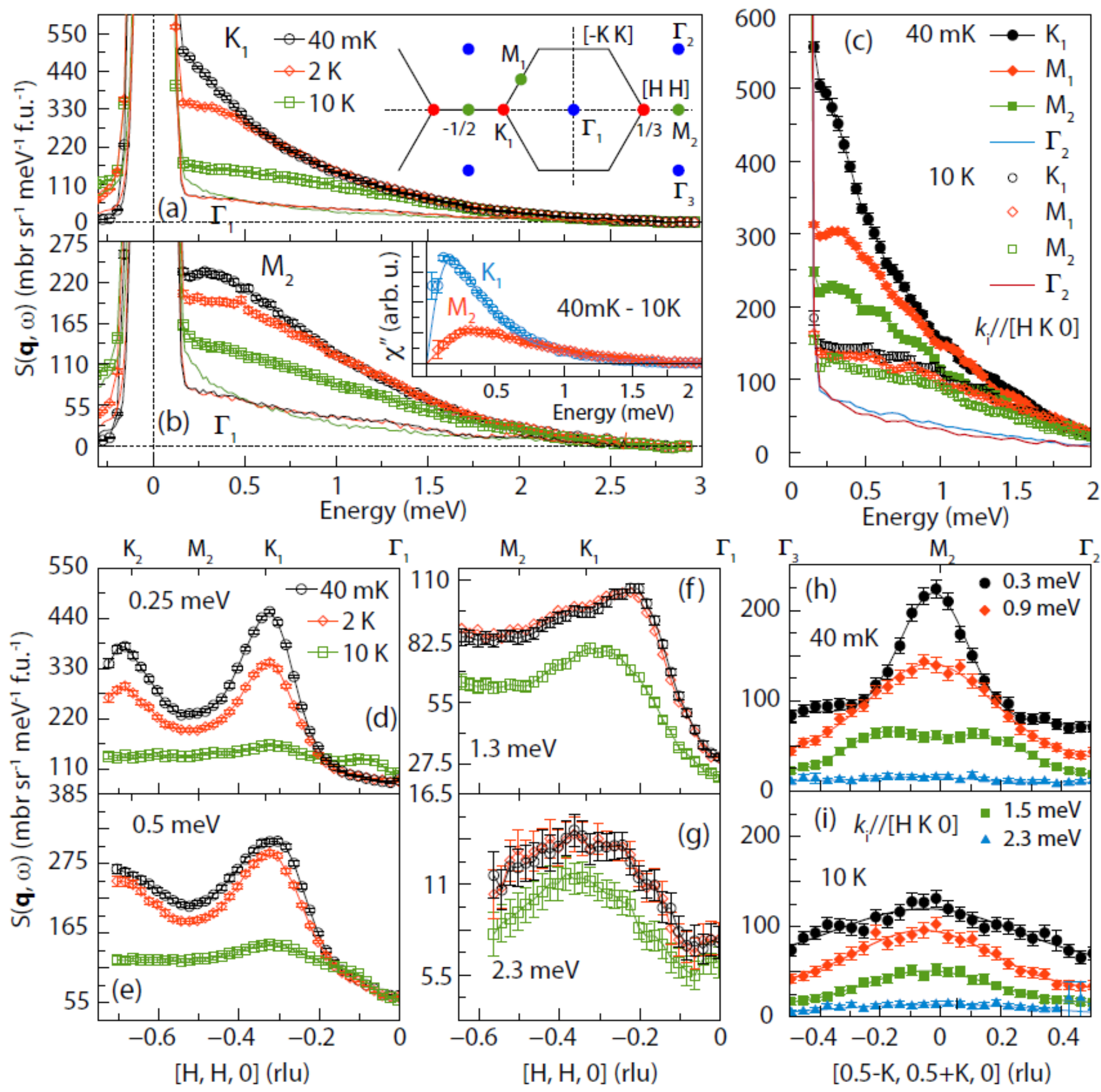

Figure 5

Wave vector dependence of spin excitations along high symmetry directions. The wave vector cuts in $(a, b, d-g)$ were measured in the $[\mathrm{H}, \mathrm{H}, \mathrm{L}]$ zone with $\mathrm{Ei}=3.32 \mathrm{meV}$, while those in $(\mathrm{c}, \mathrm{h}, \mathrm{i})$ were measured in the $[\mathrm{H}, \mathrm{K}, 0]$ plane with $\mathrm{Ei}=3.70 \mathrm{meV}$. (a) and (b) show the energy dependent scattering at $\mathrm{K} 1$ and $\mathrm{M} 1$ points measured at $\mathrm{T}=40 \mathrm{mK}$ (black circle), $2 \mathrm{~K}$ (red diamond) and $10 \mathrm{~K}$ (green square). The inset in (a) is a schematic of the reciprocal space with the $\Gamma, \mathrm{K}$ and $\mathrm{M}$ points denoted by green, red and blue dots. The black, red, and green curves are energy cuts at $\Gamma 1$. The inset of (b) shows the difference of $\chi^{\prime \prime}$ between the spectra for $\mathrm{T}=40 \mathrm{mK}$ and $10 \mathrm{~K}$ at the $\mathrm{K}$ and $\mathrm{M}$ points. The light blue and red curves are fittings of the $\chi^{\prime \prime}$ 
with a damped harmonic oscillator model.(c) shows the energy cuts at the K1, M1, M2 and $\mathrm{T} 2$. Solid symbols represent the data collected at $\mathrm{T}=40 \mathrm{mK}$ and the open symbols collected at $10 \mathrm{~K}$. The black and blue curves are energy cuts at the $\Gamma 2$ point measured at $\mathrm{T}=40 \mathrm{mK}$ and $10 \mathrm{~K}$. (d-g) Constant energy cuts along the M2-K1- $\Gamma 1$ for $\mathrm{T}=40 \mathrm{mK}, 2 \mathrm{~K}$, and $10 \mathrm{~K}$, with corresponding energy transfers marked in the panels. Constant energy cuts along the ГЗ-M2-Г2 measured at (h) $\mathrm{T}=40 \mathrm{mK}$ and (i) $10 \mathrm{~K}$. The solid curves are guides to the eyes and the error bars represent one standard deviation.

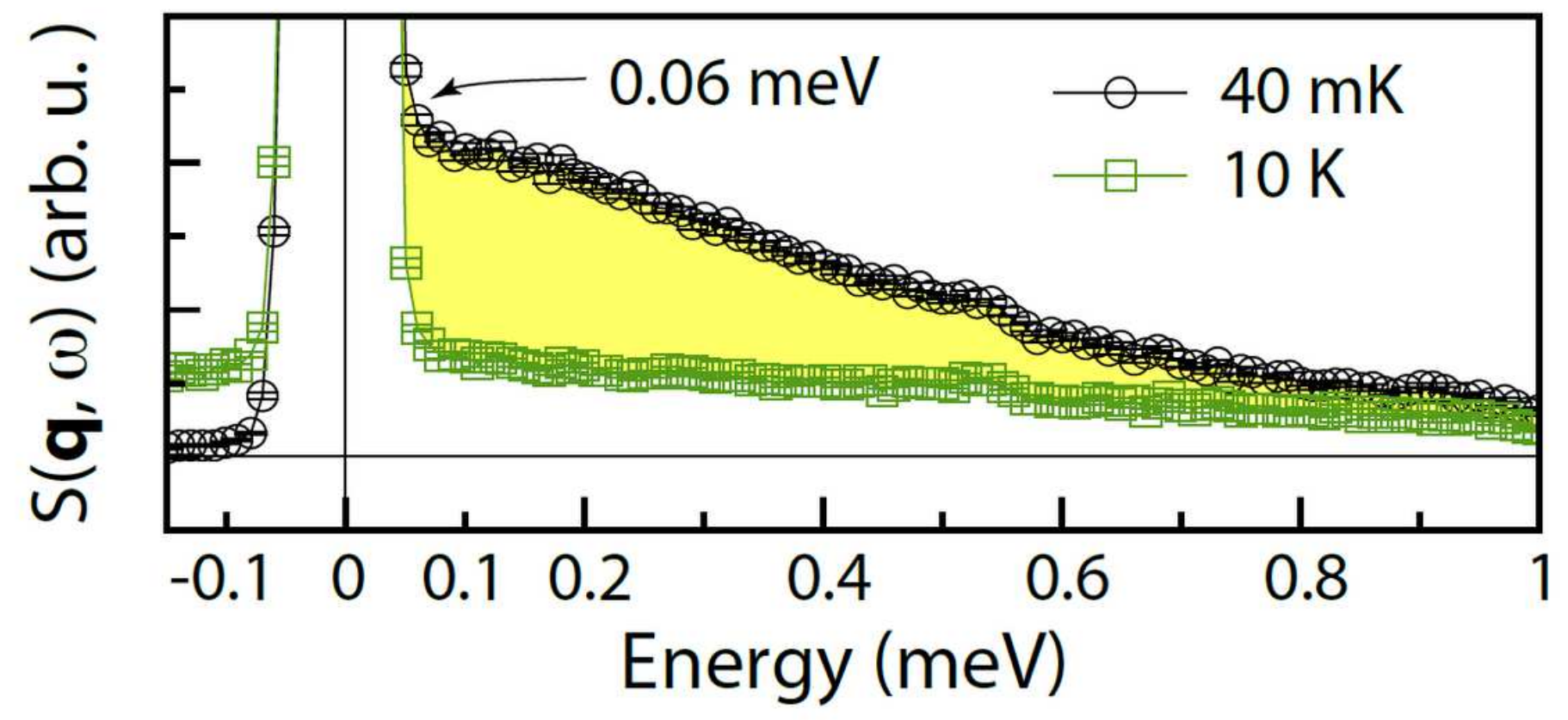

Figure 6

Spin excitation energy spectra at $\mathrm{K} 1$ position measured with $\mathrm{Ei}=1.55 \mathrm{meV}$ at $\mathrm{T}=40 \mathrm{mK}$ (red circles) and $10 \mathrm{~K}$ (green squares). The yellow shaded area marks the difference between the spectra for $\mathrm{T}=40 \mathrm{mK}$ and $10 \mathrm{~K}$. The black arrow marks the lowest energy $(0.06 \mathrm{meV})$ magnetic excitations.

\section{Supplementary Files}

This is a list of supplementary files associated with this preprint. Click to download.

- NaYbSe2SI0909.pdf 